\title{
Neuronal vector coding in spatial cognition
}

Andrej Bicanski ${ }^{\dagger}$ and Neil Burgess ${ }^{\dagger}$

Institute of Cognitive Neuroscience, University College London, London, United Kingdom

† email: a.bicanski@ucl.ac.uk; n.burgess@ucl.ac.uk 
Abstract| Several types of neurons involved in spatial navigation and memory encode the distance and direction (that is, the vector) between an agent and items in its environment. Such vectorial information provides a powerful basis for spatial cognition by representing the geometric relationships between the self and the external world. Here, we review the explicit encoding of vectorial information by neurons in and around the hippocampal formation, far from the sensory periphery. The parahippocampal, retrosplenial and parietal cortices, as well as the hippocampal formation and striatum, provide a plethora of examples of vector coding at the single neuron level. We provide a functional taxonomy of cells with vectorial receptive fields as reported in experiments and proposed in theoretical work. The responses of these neurons may provide the fundamental neural basis for the (bottom-up) representation of environmental layout and (top-down) memoryguided generation of visuo-spatial imagery and navigational planning.

\section{[H1] Introduction}

Place cells fire whenever an animal traverses a specific location in an environment (the spatial receptive field [G] of that neuron, also known as its 'place field', FIG. 1a). Since the discovery of place cells in the rat hippocampus by O'Keefe and Dostrovsky ${ }^{1}$, researchers have uncovered a multitude of additional spatially-selective cell types in rodents: that is, neurons whose receptive fields reference some aspect of an organism's location, state of motion, pose or its relationship to environmental features (such as boundaries, landmarks and other objects). The spatial receptive fields of some of these cells correspond to vectors, indicating the distance and direction in space (relative to the animal's current location) within which the presence of an environmental feature will drive the neuron to fire. Such 'vectorial codes' for space have received comparatively less attention than the coding performed by place cells or other well-known spatially-selective cell types, such as grid cells [G] ${ }^{2}$ (FIG. 1a) and head direction cells [G] ${ }^{3,4}$.

Vectorial codes for space are expressed by boundary vector cells $\mathrm{s}^{5,6}$, border cells $\mathrm{s}^{7,8}$, landmark vector cells $\mathrm{s}^{9}$ and object vector cells ${ }^{10}$ in allocentric (that is, world-centered) coordinates. Just like place fields, the receptive fields of these vector-coding cells do not reflect the orientation of the animal but do rotate together with the prominent environmental features that control head direction cell firing ${ }^{11}$. Egocentric counterparts of some of these cells — in which the direction of receptive fields are referenced relative to the facing direction of the agent - have also been found ${ }^{12-15}$, as well as cells that exhibit head direction-modulated boundary responses ${ }^{16,17}$. 
The vectorial properties of boundary, object and landmark vector cells derive from receptive fields that reference locations outside an agent's current position (FIG. 1b). Rather than being centred on the location of the organism (like place fields FIG. 1a) these receptive fields can be thought of as covering locations around an agent. These cell types may thus form a neural representation of the structure of the world 'out there'; that is, the geometric configuration of landmarks, borders, and objects relative to the agent. As such, these cell types differ in several ways from place cells, grid cells and head direction cells. For instance, the responses of place, grid and head-direction cells are often robust with regards to the removal of individual environmental cues and typically reflect the spatial configuration of multiple environmental features, rather than the presence of a single feature ${ }^{18,19}$. Vector coding neurons, on the other hand, represent the presence of an environmental feature at a specific location relative to the agent. If their receptive fields cover space uniformly, a population of these neurons can therefore represent the layout of environmental features around an agent $^{20,21}$. In many cases such cells code for the presence of any object at the location of their receptive field, rather than coding for the sensory characteristics of a specific item in the receptive field $^{6,10}$.

If all distances and directions are equally represented, the receptive fields of multiple vector coding cells may be seen as forming a grid that is anchored to the agent's position and translates with the agent (FIG. 1b-d). In the case of allocentric responses, the grid does not rotate with the agent (as allocentric North is independent of the animal's orientation), whereas, for egocentric vectorial responses, the grid translates and rotates with the agent (as egocentric 'ahead' depends on the current heading direction).

In this Review, we provide a taxonomy of the growing number of cells with vectorial receptive fields in terms of their response properties (Table 1). Since most of these cell types have been characterised in rodents we focus on the rodent literature in the review, but relate these findings to other species (including humans) where appropriate. We begin by reviewing allocentric vector coding neurons and their relationship to place cells. We then discuss their more recently discovered egocentric analogues and their relationship to goal vector coding in flying bats ${ }^{22}$. Finally, we proceed to review theoretical models that may explain how the egocentric and allocentric cell types interact to support spatial cognition, before considering open questions in the field.

\section{[H1] Allocentric vector coding}


Boundary vector cells were first proposed on a theoretical level, as the cells that provide inputs to place cells. How else to determine one's (absolute) location in a given environment if not in reference to external landmarks? Finding oneself 2 meters from the North wall, and 5 meters from the West wall within a simple square room specifies one's location perfectly. Experiments demonstrating the influences of environmental deformations (changes in environment size and shape) on place fields ${ }^{23}$ led to the development of the boundary vector cell model of place cell firing ${ }^{24,25}$. This model showed that a simple thresholded sum of boundary vector cell responses can model the characteristics of place fields, and the effects of changes to the geometric properties of simple environments. Such a model of self-localisation is consistent with McNaughton and colleagues' suggestion that place cells may represent vectors to specific landmarks ${ }^{26}$. It also lies at the core of models proposing that spatial cognition [G] is supported by translations between egocentric and allocentric vectorial responses ${ }^{20,21,27}$ (discussed below), which provide a framework for understanding the various vectorial cell types that have been identified.

Boundary vector cells were first experimentally identified in the subicular complex of rats ${ }^{5}$ and their properties were systematically examined in subsequent studies ${ }^{6,27}$. Boundary vector cells fire when an animal is at a given distance and direction from any boundary, including barriers inserted into an experimental arena (FIG. 2a), rather than signalling the characteristics of specific features of the boundary (such as a particular piece of wood) ${ }^{6}$. This allows the boundary vector cell model of place cell firing to account for the doubling of place fields ${ }^{5}$ in response to inserted barriers. That is, the firing of a population of boundary vector cells can cause multiple peaks in the firing of place cells that result from summing boundary vector cells ${ }^{28}$ with similar activations in multiple locations (FIG. 2a). Similarly, more complex, repeating boundary configurations within the same environment may yield multiple place fields. This property has been impressively replicated in extensive simulations of the boundary vector cell model ${ }^{29}$, which largely reproduced the firing of experimentally recorded place cells in a multitude of different (and visually repeating) environmental configurations. Secondary place fields have been shown to attenuate with experience ${ }^{30}$, which can be modelled with the inclusion of a BCM learning rule ${ }^{31}$ acting between boundary vector cells and place cells ${ }^{5}$. According to this model, the allocentric nature of boundary vector cells also causes place cell firing to be independent of the animal's orientation in open fields.

Experiments further revealed that boundary vector cells are similarly responsive to vertical walls made of different materials and to the drop at the edges of a raised platform ${ }^{6}$. Small, traversable gaps in the floor of a recording arena also elicited boundary vector cell responses ${ }^{6}$ (FIG. 2b). 
Moreover, these responses are present from first exposure to the environment and are stable over time $^{27}$. Different boundary vector cells have receptive fields tuned to different distances and directions $^{6}$, suggesting that a population of boundary vector cells does indeed provide a grid of receptive fields covering space around the organism. However, the receptive field size appears to increase with distance, leading to broader bands of activity in firing rate maps $[\mathbf{G}]$ and thus a coarser representation of more distant environmental features ${ }^{6}$ (FIG. 2c; FIG. 1). Though the relative distribution of boundary vector cells with distal versus proximal receptive fields has not been systematically quantified, both experimental data ${ }^{6}$ and models ${ }^{24}$ suggest that proximal fields are likely to be more prevalent. The range of encoded distances (in all allocentric directions) constitutes the main distinguishing feature between boundary vector cells and border cells, another type of vector coding cell that is found in the medial entorhinal cortex ${ }^{7,8}$. Border cells (FIG. 2d) are defined by their propensity to respond only to proximate boundaries (that is, those in whisking [G] range of rodents). It has been hypothesised that border cells may serve in immediate obstacle detection $^{32}$ or in anchoring grid cell firing to environmental borders ${ }^{33,34}$. Boundary vector cells have also been proposed to support these and other aspects of spatial cognition $20,21,35,36$. Another important distinction between border cells and other vector coding cells is that border cells only respond to a boundary that blocks an animal's path ${ }^{10}$. Boundary vector cells (and object vector cells, discussed in more detail below) can be driven by features that do not present navigational impediments (specifically, traversable gaps in the case of boundary vector cells ${ }^{6}$ and elevated objects in the case of object vector cells ${ }^{10}$ ).

Self-localization based on sensory signals is a necessary complement to self-localization via the path integration [G] inputs to place cells that are thought to be mediated by grid cells ${ }^{37-39}$. The fact that boundary vector cells are sensitive to boundaries behind an animal suggests that these cells do not rely solely on sensory perception. That is, they incorporate a mnemonic component.

Nevertheless, the boundary vector cell model of place cell firing suggests that, by driving individual place cells, boundary vector cells accomplish self-localization with respect to extended environmental features (such as the walls in a room) or large landmarks (such as the buildings around a square).

One potential challenge to this model of the environmental inputs to place cells concerns the anatomical distribution of boundary vector cells. Large numbers of boundary vector cells have been reported in the subiculum ( $24 \%$ of 186 recorded subicular cells ${ }^{6}$ ), but only small numbers have been reported in the medial entorhinal cortex ${ }^{10,40}$, where object vector cells (see below) and border cells 
are more plentiful (for example, $14.7 \%$ of 1100 recorded cells in medial entorhinal cortex were classified as object vector cells in a recent study ${ }^{10}$ ). The subiculum is traditionally thought of as an output region of the hippocampal formation, whereas medial entorhinal cortex superficial layers are a major input, suggesting that subicular boundary vector cells are 'outputs' rather than 'inputs' for hippocampal place cells. Three possible solutions to this puzzle have been proposed. First, neurons in subiculum may project into hippocampus. Indeed, there is now significant evidence for direct projections from the subiculum to the hippocampal subfield $C A 1^{41-50}$. These projections are capable of affecting place cell firing, and are of a similar strength to the projections from the entorhinal cortex to $\mathrm{CA}^{48}$. However, this leaves open the question of the source of environmental input to the hippocampal subfield CA3. Second, although only small numbers of boundary vector cells have been reported in medial entorhinal cortex so far, there may be enough to provide environmental input to place cells, given that they combine combinatorially to determine place fields ${ }^{24}$. Finally, it is possible that border or object vector cells in the medial entorhinal cortex provide environmental inputs that anchor grid cells to the environment and grid cells then drive place cell firing ${ }^{34}$. However, there are reasons to doubt that place firing relies entirely on grid cells, given their different developmental trajectories ${ }^{22,51}$ and differential dependence on environmental and selfmotion information ${ }^{52}$.

Theoretical boundary vector cells were conceived to be capable of responding to environmental features of any size, with large extended features causing more firing than small discrete ones (with large features occupying the receptive field at more locations ${ }^{54}$ ). More recently, we suggested that a separate class of cells — object vector cells - may employ the same receptive field structure as boundary vector cells, but respond specifically to discrete objects and not to the surrounding boundaries $^{21}$ (FIG. 2e-f). This suggestion arose from the idea that, while boundary vector cells enable self-localisation relative to the (stable) layout of extended environmental features, spatial memory also requires neurons that represent the locations of smaller, potentially mobile or more ephemeral objects that may be less-well suited to determine location of the exploring agent ${ }^{21}$. These theoretical cells were also inspired by the landmark vector cells previously discovered in the hippocampus ${ }^{9}$ (discussed see below).

Independently of theoretical considerations, object vector cells were discovered experimentally in the medial entorhinal cortex ${ }^{10}$ (FIG. 2e) and shown to respond to objects at given allocentric distances and directions. Characterization of these cells revealed that they show little modulation by head direction (similar to boundary vector cells) and respond to objects at a range of distances ${ }^{10}$ 
(FIG. 2h). Although most object vector cells fired when the distance between the agent and object was below $30 \mathrm{~cm}$, some object vector cells with more distant receptive fields (up to $50 \mathrm{~cm}$ ) were also reported ${ }^{10}$. This contrasts with border cells which fire only when the agent is close to a boundary ${ }^{7}$. Importantly, concurrently recorded object vector cells maintain their relative vectorial differences (angle and distance from objects) when an animal moves to a new environment (FIG. 2i). This finding is reminiscent of reports that concurrently recorded head direction cells maintain their relative angular distance in different environments ${ }^{11}$. Moreover, it corroborates the notion that a population of object vector cells instantiates a fixed grid of receptive fields (which is centred on the animal but does not rotate with it in the case of allocentric cells, FIG. 1). The orientation of the grid may change between environments (depending on the environmental features determining the sense of direction that is reflected by head direction cells), but the relative differences between cells are preserved. Interestingly, as noted above, object vector cells continue to respond to an object even if it is suspended above the ground so that the animal can walk underneath, in contrast to border cells which do not fire in response to a suspended border ${ }^{10}$ (FIG. 2i).

Object vector cells fire for small within-arena objects but not to the boundaries of the environment ${ }^{10}$. Unless there are other factors differentiating internal objects from surrounding barriers, such as novelty or permanence, this suggests that object vector cells may have on-centeroff-surround receptive fields, in which the activity of the neuron is only fully driven when the excitatory influence of a (small) central object is not cancelled out by inhibitory influences on either side (FIG. 2k). This receptive field structure must apply also to elevated objects, to explain object vector cell responses (FIG. 2i). The receptive field structure of boundary vector cells, on the other hand, would be lacking this inhibitory surround. Whether boundary vector cells also respond to elevated objects has not yet been tested. Similarly, it remains to be determined whether object vector cells respond to drops, or to gaps in the floor (FIG. 2b, also see below).

As noted above, a similar type of allocentric vectorial information appears to be represented in the landmark vector cells that have been recorded in small numbers in the hippocampus ${ }^{9}$ (FIG. 2j). Landmark and object vector cells differ in that landmark vector cell firing is modulated by the object's identity, while object vector cell firing is not (just as boundary vector cell firing is not modulated by the identity of the boundary) ${ }^{10}$. It has been suggested that the modulation of hippocampal landmark vector cell activity by object identity might indicate that they receive input from both object vector cells in the medial entorhinal cortex and from cells that represent object identity in lateral entorhinal cortex ${ }^{55}$. However, landmark vector cell firing appears to be modulated 
by more than one object at a time, with the identity of the objects sometimes changing over time, precluding a simple account of their firing selectivity at this moment. In addition, landmark vector coding in the hippocampus appears to be experience-dependent. A subset of recorded landmark vector cells developed new firing fields [G] when an object or landmark was moved during a recording session. However, only in some cases, these fields had similar distance and direction tuning as the initial field, as would be expected from object vector cells ${ }^{9}$. Some landmark vector cells increased their firing (when objects moved to new locations) at locations where the objects used to be ${ }^{9}$ (also see below). These cells might therefore be involved in memory for an object's identity and location.

In summary, there is considerable evidence that the location of both extended environmental features and smaller objects within that environment are coded for by populations of neurons whose allocentric vectorial receptive fields form a grid that covers the space around an organism. The recording of multiple firing fields in object vector cells and in boundary vector cell experiments with inserted boundaries, ${ }^{6}$ suggest that these allocentric receptive fields are indeed anchored to the agent and translate with it, coding for the presence (but not identity) of objects or landmarks relative to the organism.

\section{[H1] Egocentric vector coding}

The allocentric neural responses described above are not coded in the reference frame [G] (that is the coordinate system) of any sensory receptor, being independent of the animal's orientation. In order to be able to convey environmental information (such as vectors to objects or boundaries), the response properties of allocentric neurons must be derived from neurons whose responses are coded in the reference frame of sensory perception (that is ahead, left and right rather than North, South, East and West). Therefore, neurons with egocentric (body or head-centred), rather than allocentric, vectorial responses must be present upstream of the allocentric representations. Recent experimental findings have demonstrated the presence of such egocentric boundary coding neurons in the striatum $^{13}$, retrosplenial cortex ${ }^{14,15}$, lateral entorhinal cortex ${ }^{12}$ and postrhinal cortex ${ }^{17}$. These cells fire in response to boundaries ahead of or to the left or right of the rodent. In an allocentric frame of reference these cells would appear to respond to all walls of an environment (rather than, say, only the North wall) because they fire every time a wall is ahead (for example) of the animal (FIG. 3a). Hinman and colleagues designated these cells as 'egocentric boundary cells'13. 
Similar to boundary vector cells, egocentric boundary neurons in the dorso-medial striatum ${ }^{13}$ show an increase in receptive field size with tuning distance from the animal (FIG. 3b). The cells fire irrespective of the shape, visual appearance or allocentric orientation of the environment and are stable across repeated visits to the same environment ${ }^{13}$. This suggests that these cells code for geometric environmental features, irrespective of low-level sensory characteristics, in egocentric coordinates. Importantly, despite their egocentric tuning, egocentric boundary cells in the striatum are not purely sensory or perceptual, since some cells fire also for boundaries behind the animal, suggesting a mnemonic component. The striatal egocentric boundary neurons were further reported to exhibit a curious trimodal distribution of preferred distances and a bi-modal distribution of preferred directions, with most cells having a preferred direction 90 degrees clockwise or counterclockwise of the animal's head direction. This distribution is not evident in the retrosplenial cortex egocentric boundary cells discussed below.

Why would egocentric boundary cells be present in the striatum? The theoretical models of spatial cognition which predicted egocentric boundary cells ${ }^{20,21,35,36}$ suggest that they form the principal inputs to boundary vector cells (see below), but do not address striatal function. However, the striatum is heavily implicated in reinforcement learning of the mapping from egocentric sensory inputs to motor outputs which are also coded in an egocentric (body-centered) frame of reference, during cue-based navigation ${ }^{56}$. In experiments in which animals have been overtrained to use environmental cues to find a goal show that they use an egocentric motor-turn strategy (e.g. always turning left) that depends on the striatum ${ }^{57}$. This provides a tentative explanation for egocentric boundary coding in the striatum but does not suggest that egocentric boundary responses necessarily originate there (see below).

Egocentric boundary cells have also been reported in the dysgranular retrosplenial cortex ${ }^{14}$. These cells do not exhibit any particular topography within the dysgranular retrosplenial cortex and a subpopulation of them are modulated by theta oscillations [G]. Unlike boundary vector cells, some egocentric boundary cells in retrosplenial cortex (specifically those with proximal receptive fields) do not exhibit firing in arenas in which the walls have been removed (FIG. 3c). Their directional preference appears to be lateralized, with preferential tuning for borders left of the animal in the right hemisphere and vice versa (BOX 1). Like striatal egocentric boundary cells, the firing of these cells is context invariant (FIG. 3d), being independent of self-motion and the shape or orientation of the local environment relative to distal cues (that is, the laboratory frame). These findings are again consistent with the notion that these cells code for the geometric layout of environmental 
features irrespective of their low-level sensory characteristics. The possibility that egocentric boundary cell responses are better described as coding for an egocentric vector to the center of the recording arena has also been excluded ${ }^{14}$. This corroborates the idea that the receptive fields of egocentric boundary cells are anchored to the animal (FIG. 1b-c) just like those of boundary vector cells, but with one crucial difference: the receptive fields of egocentric boundary cells rotate with the agent (they are egocentric). In a subset of animals, egocentric boundary coding neurons were also found in the secondary motor cortex and the posterior parietal cortex ${ }^{14}$.

Another recent study also report the presence of egocentric border cells in the restrosplenial cortex ${ }^{15}$. The cells fired in response to newly inserted barriers (but not objects), and retained their firing in darkness. Corroborating the findings of Alexander and colleagues ${ }^{14}$, the directional preference of these cells appears to be lateralized. These cells also fired in response to environmental edges without walls, and their response properties depend on intact entorhinal afferents. Egocentric boundary cells_ — including cells that fire preferentially for boundaries behind the animal — have also been identified in postrhinal cortex ${ }^{17}$. However, while egocentric boundary cells with distant receptive fields were reported in this region, a markedly higher proportion of cells fired for boundaries at close range.

It is often assumed that allocentric coding dominates within the hippocampal formation (including the entorhinal cortex). Importantly, however, neural representations of the egocentric bearing of boundaries and objects have recently been reported in the lateral entorhinal cortex ${ }^{12}$ (FIG. 3e). In this study, some lateral entorhinal cells were shown to fire when a boundary or object is found at a given egocentric bearing, and that bearing was stable within a session and consistent between different recording arenas (small and large boxes). A significant percentage (approx. 20\%) of recorded cells was also tuned to the distance of the nearest boundary or object ${ }^{12}$. Thus, these cells qualify as vector coding cells (encoding direction and distance). However, it was reported that, between sessions with different behavioral goals. The number of cells that represented the standard versus the displaced goal locations changed significantly ${ }^{12}$, possibly suggesting the coding of behaviorally relevant objects or goal locations. This behavioural modulation is subtly different from the predicted properties of egocentric object coding neurons ${ }^{21}$ (see below). The involvement of the lateral entorhinal cortex in object processing ${ }^{58}$ also suggests that the sensory features of objects or boundaries may contribute to driving these lateral entorhinal cells. 
Recent papers have reported that there is also an egocentric vectorial code for abstract locations within an environment. Populations of cells in the postrhinal cortex (the rodent homologue of parahippocampal cortex, see below) have been reported to code for the egocentric bearing and distance of the centre of a square arena ${ }^{59}$. A subset of these cells is also modulated by allocentric head direction (FIG. 3f). Allocentric positional information - present in downstream structures in the form of place cells in hippocampus and grid cells in medial entorhinal cortex — can be decoded from the responses of these cells, which have been termed 'egocentric center-bearing' and 'distance' cells. The bearing and (absolute) distance correlates of these cells persist in smaller or rotated square environments. In a related article, so-called 'anchor cells' have been reported in the human parahippocampal cortex, recorded from epilepsy patients with implanted depth electrodes ${ }^{60}$. These cells are tuned to an egocentric bearing to (anchor) points distributed across the entire recording arena (not just its center). A subset of these cells also shows distance tuning. Though anchor points are distributed throughout the virtual recording arena there is a significant concentration of anchor points in the center, raising the possibility that egocentric centerbearing/distance cells ${ }^{59}$ constitute a subset of anchor cells. The distribution of anchor points also resembles the distribution of maximum firing locations of egocentric bearing cells in Wang et al. ${ }^{12}$ (discussed in the preceding paragraph), clustering around the center but distributed across the entire environment. Anchor points in the center of a radially symmetric environment are inherently ambiguous as to whether they reflect egocentric bearing relative to the center or to the nearest boundary. Wang et al. ${ }^{12}$ describe their results in terms of boundary coding (acknowledging this ambiguity). Anchor cells may also be related to hippocampal cells that reference remote locations (even beyond an open field enclosure), as reported by Jercog and colleagues. ${ }^{61}$.

Finally, angle-to-goal and distance-to-goal cells have been identified in the hippocampal subfield CA1 of bats ${ }^{22}$. These cells fired when a hidden goal platform was at a specific egocentric angle relative to the direction of flight. Cells tuned for preferred goal directions ahead were overrepresented within the population of these cells, compared to those tuned to other possible directions. A subset of these cells had additional spatial tuning, meaning they signalled the egocentric direction and distance of the goal location in the environment. The tuning of these CA1 cells does not persist across sessions or within session when the navigational goal changes. Hence, although these cells are reminiscent of egocentric boundary cells in rodents, it appears that they are dedicated to representing a specific behaviorally relevant location (the goal in a navigational task) rather than signalling the presence of arbitrary environmental features. 
At a theoretical level, the egocentric coding of boundaries and landmarks was considered repeatedly prior to the experimental confirmation of egocentric boundary responses. In one early model of place cells ${ }^{62}$ an array of stimuli was distributed around the perimeter of a simulated environment, and inputs to the entorhinal cortex coded for either the distance from the stimuli (an orientation invariant signal) or the egocentric bearing of the stimuli (an egocentric signal). The activity patterns in the entorhinal cortex were then generated by competitive learning [G] and yielded a conjunctive representation of bearing and distance. The activity patterns in entorhinal cortex were in turn classified by hippocampal cells via competitive learning, yielding place cell responses. Thus, the model associated all possible egocentric sensory snapshots (of stimulus distance and bearing) with a single cell whose place field was consistent with those views, consistent with the notion of egocentric sensory snapshots for self-localization, outlined later by McNaughton and colleagues ${ }^{26}$.

Mirroring the account of boundary vector cells given above, egocentric boundary vector coding neurons were predicted in a series of subsequent models $\mathrm{s}^{20,21,35}$ both as the sensory inputs to boundary vector cells and as an egocentric output of the hippocampal system supporting imagery and planning. Building on these models, egocentric object-coding neurons were predicted recently (alongside allocentric object vector cells ${ }^{21}$ ) and show similarities to concurrently discovered egocentric object bearing neurons in lateral entorhinal cells ${ }^{12}$. This family of computational models avoids some limitations of models of self-localization based purely on associating large numbers of egocentric sensory snapshots with a given location. For example, the interference that can occur between visually similar (egocentric) views obtained when an individual is located in different parts of the environment. In addition, memorizing many sensory snapshots (for different egocentric orientations) at a single location is inefficient, compared to memorizing a single allocentric representation from which egocentric views can be reconstructed (see below). On an empirical level, the phenomenon of representational neglect due to parietal/retrosplenial cortex lesions (BOX 1), suggests that recall of egocentric representations depends on underlying viewpoint-invariant (that is, allocentric) neural representations that remain intact when their egocentric counterparts are (partially) damaged by a lesion. Connecting egocentric representations of landmarks with allocentric representations of landmarks through a coordinate transformation solves these conceptual issues and provides a neural-level model of representational neglect ${ }^{20,63}$. This type of model has received recent support through the report of boundary-by-head direction coding neurons in rodents ${ }^{16,17}$ (discussed below).

\section{[H1] Reference frame transformations}


If egocentric and allocentric boundary and object vector cells convey information about the locations of environmental features, then there must be a sensory origin of these signals (see below). Thus far, the term 'egocentric' has been synonymous with a head-centered representation. However, the first reference frame of visual signals is given by the retina. This signal must then be transformed into a head-centered reference frame. This transformation has been subject to extensive modelling, following the discovery of so-called gain-field neurons $[\mathbf{G}]$ in the monkey parietal cortex $^{63-67}$.

Gain field neurons code for a combination of variables. For example, they may respond to both the position of the eye and the eye-centered (retinal) location of the stimulus, with the eye-position modulating the response to the retinal location (changing the 'gain' of that response). If the eyes move but the head stays stationary the stimulus position relative to the head has not changed, but the stimulus position on the retina has. Thus, a different combination of eye-position and stimulus location on the retina, coded for by a different gain field neuron, will map onto the same craniotopic neuron. This way, the retinal location of a stimulus can be mapped onto its angle relative to the head via the eye-position signal, with gain field neurons implementing a mechanism for reference frame transformations ${ }^{63,66}$. Different reference frame transformations can work in a similar way and may be chained together ${ }^{68}$ or act in parallel ${ }^{63}$ (though the former requires only a linear increase in the number of neurons with the number of frames of reference, which may be preferable). For example, one could transform retinal location into head-centered location and from there into trunk-centered coordinates for grasping, while - at the same time - using the head-centered representation as input for the egocentric-allocentric transformation described in detail below.

In the context of representation and memory of environmental layout, a reference frame transformation takes egocentric (head-centered) boundary or object directions and distances as input and produces allocentric boundary or object distances and directions (FIG. 4). Here head direction plays the role of the gain signal. That is, an intermediate layer of head-direction modulated boundary vector neurons (the gain field neurons in this context), allow allocentric boundary or object vector cell responses to be generated from egocentric inputs, thus enabling allocentric encoding of an environment ${ }^{20,21,35}$. Equally, the reverse flow of information (via reciprocal connections) can reconstruct egocentric boundary and object vector responses from stored allocentric representations during recall or imagery ${ }^{20,21,35}$. The gain-field neurons which map egocentric boundary distances and directions to allocentric boundary distances and directions should respond to conjunctions of boundary distance and head direction (see also Ref. ${ }^{69-71}$ ). It is 
important to note that the egocentric-allocentric transformation mechanism does not require a very fine granularity of the gain signal (in this case, the head direction). Models $\mathrm{s}^{20,21,35}$ show that as few as 20 discrete layers, each maximally modulated by one of 20 (equally spaced) head directions guarantees enough overlap (given a Gaussian head direction signal) to successful interpolate between maximally effective head directions.

Recent studies reported the identification of cells with the predicted vectorial receptive fields that are modulated by the head direction of the animal ${ }^{16,17}$ (FIG. 4b). Because these cells (like those in the transformation circuit described above) fire for only one head direction and one boundary direction it is impossible to characterise the boundary-related information that they encode as egocentric or allocentric. The cells fire irrespective of environmental shape ${ }^{17}$, and collectively, they code for boundaries in all directions relative to the animal, (including behind it) ${ }^{17}$, again suggesting that their firing patterns are not driven purely by sensory input (though directions ahead of the animal were preferentially represented). The cells were mainly found in postrhinal and medial entorhinal cortices, subiculum, and parasubiculum ${ }^{17}$. A different study also reported similar cells in the postsubiculum ${ }^{16}$. Theoretical models had suggested retrosplenial or parietal cortices ${ }^{20,21,63}$ following primate recordings ${ }^{65}$ and human neuroimaging studies ${ }^{68,72-74}$. It remains to be seen whether boundary-by-head direction coding in rodents is localised in the extended network of structures implicated by a recent study ${ }^{17}$ (also see Ref. ${ }^{75}$ ). Nevertheless, these findings ${ }^{16,17}$ provide strong experimental support for the predicted gain-field mechanism of egocentric-allocentric reference frame transformations for boundary coding that was previously explored in multiple theoretical models $\mathrm{s}^{20,21,35,36}$, and show how it can be applied specifically border cells ${ }^{17}$.

\section{[H1] From single cells to spatial memory}

Given that hippocampal place cells represent an agent's location, head direction cells an agent's orientation, and vector coding neurons the structure of the world 'out there' as viewed from a given location, how may spatial cognition emerge from the interplay of these representations? Theoretical models $\mathrm{s}^{20,21,35}$ have suggested a systems-level account of spatial cognition, integrating the functions of known spatially-selective cell types and predicting others. In this family of models the locations of scene elements (boundaries, objects and landmarks) are represented in the firing of egocentric boundary and object vector cells, which are transformed into allocentric boundary and object vector cells by the gain field circuit (see above). To bind the elements of a scene (as perceived at a given location), it is suggested that synaptic connections between the allocentric vectorial representations and a 'binding cell' are formed. Since the momentary activity profile across egocentric cells (and 
hence their allocentric counterparts) is unique for the given location, the binding cells (in hippocampus) exhibit the firing characteristics of place cells (location tuning, see also Ref. ${ }^{71}$ ), consistent with the boundary vector cell model of place firing (see BOX 1 for the relationship to human spatial cognition).

According to these models, the identities of scene elements are coded by separate populations of ventral visual stream neurons, potentially in the perirhinal cortex. Thus, the locations of objects 'out there' and their identities are coded separately, but are bound together via Hebbian learning [G] during exploration. Together, place cells, perirhinal neurons and the various allocentric vector coding neurons form a continuous attractor (a network whose pattern of connections constrains the neural activity to a continuous set of locally stable patterns) describing the layout of an environment. In these models, egocentric cells were suggested to reside in parietal areas ${ }^{14}$ — the precuneus in particular - and to represent the neural correlate of an agent's egocentric point of view (what is ahead, left, right, and so on). Within this framework, perceiving the local layout of scene (via egocentric representations) allows an agent to self-localize by activating a corresponding allocentric vectorial code (via the reference frame transformation), which in turn drives corresponding place cells. The reverse flow of information, from allocentric representations (place cells, object and boundary vector cells) through the gain-field circuit, driving egocentric representations, provides an account of memory recall in the form of visuo-spatial imagery. That is, the representations of scene elements 'out there' in egocentric terms are re-constructed from the underlying allocentric representations in the medial temporal lobe via top-down re-instatement. FIG. 5a depicts a high-level schematic of this theoretical framework.

This neural-level account of memory recall shows how egocentric and allocentric vectorial neural representations could interact and allows for several predictions. For instance, recalling a scene with scene elements (boundaries or objects) that have recently been removed would lead to activity traces for locations consistent with the position of those absent elements ${ }^{21}$ (FIG. 5b-c). Such 'trace' responses may even appear in nominally non-spatial cells that normally code for the identity of objects or specific sensory features, as a result of their association with location ${ }^{21}$. Non-vectorial trace responses near object locations have been reported ${ }^{9,76-79}$ (FIG 5d, also see Ref. $^{80}$ ). Vectorial trace responses have recently been reported in the rodent subiculum ${ }^{81}$. These cells fire in response to environmental features at a certain allocentric distance and direction, including extended boundaries and smaller objects (i.e., similar to boundary vector cells in theoretical models). Most importantly, these cells also fire for the same objects after they are removed (FIG. 5b-c) $)^{21}$. These 
cells have been termed 'trace vector cells' ${ }^{81}$, and they appear to be distinct from non-trace vector cells, being found in the distal and proximal subiculum, respectively. This may suggest that distinct sub-populations of vectorial neurons code for perceptual (non-trace cells) versus mnemonic aspects (vector trace cells) of scene representation (FIG. 4a; FIG. 5a-c).

\section{[H1] Open Questions}

\section{[H2] The reference frame transformation}

The theoretical framework outlined above $e^{20,21,35,63}$ proposes the presence of a gain-field mechanism supporting translation between reference frames. The neural implementation of this mechanism has been tentatively identified in neuroimaging studies suggesting that successive translations from eyecentred to head-centered and head-centered to body- or world-centered representations are reflected in the swath of activity running between the parahippocampus and precuneus via the retrosplenial cortex in spatial memory tasks ${ }^{68}$. In particular, activity in the retrosplenial cortex has been linked to tasks requiring translation between allocentric and head-centered frames ${ }^{72}$. However, posterior parietal areas have also been identified as being associated with this function in primate recordings of gain-field neurons ${ }^{65,82}$ and human neuroimaging ${ }^{68}$.

As described above, a recent study in rats found head direction-modulated boundary responses in the para-subiculum, the postrhinal cortex and the medial entorhinal cortex ${ }^{17}$, painting the picture of a distributed network of interconnected regions with egocentric vectorial representations. A recent review of the literature similarly suggest a gradient of egocentric-allocentric processing from the parietal cortex to the retrosplenial cortex ${ }^{83}$. The extent of the reference transformation circuitry remains to be determined. However, in rodents, strong associational input to the postrhinal cortex arises in the posterior parietal cortex and dorsal retrosplenial cortex ${ }^{84}$ and the postrhinal cortex is thought to be in part homologous to the parahippocampal place area $\left(\mathrm{PPA}^{85}\right)$ in humans. This suggests some correspondence between model predictions (PPA and RSC in humans) and experimental findings (POR in rodents) regarding the location of the reference frame transformation.

Future work should clarify how the egocentric-allocentric translation of vectorial codes emphasised here relates to the rich surrounding literature on parietal and retrosplenial functions in spatial navigation, including trajectory ${ }^{86,87}$ and landmark coding ${ }^{83,88}$.

\section{[H2] Sensory input underlying egocentric coding}


The responses of egocentric boundary cells ${ }^{13-15,17}$ suggest that, in rodents, the neural correlates of the arrangement of objects in three dimensional space (at a sensory level) must be computed by the visual system prior to or within the parietal and retrosplenial cortices. However, responses to boundaries behind the animal and the presence of 'trace' firing in allocentric representations, raise the question of whether or not vectorial responses to distant objects are purely visual (for example, audition might be used to assess distance) or even purely sensory. Recording such responses in darkness would constitute an interesting first step towards a better understanding of the responses of these neurons.

It is also unclear how and where along the dorsal visual processing stream distance estimates are combined with angular bearing to produce vectorial coding. Compared to primates, rodents have comparatively poor vision with limited overlap (approx. 60 degrees) of left and right visual fields, meaning that depth cues may be harder to come by in these species. Thus, particularly for rodents, it is unclear how the distance tuning of receptive fields several body-lengths from the animal (up to 60 $\mathrm{cm}^{12}$ ) might be established. While the visual systems of primates and rodents differ substantially, recent findings provide evidence for (allocentric) boundary coding in primates ${ }^{89,90}$. Furthermore, parietal and frontal eye field neglect reported in rats ${ }^{91}$ (BOX 1) might reflect homologous functions.

In primates, the precuneus, (on the medial surface of Brodman area 7 in the parietal lobe) was suggested to hold the egocentric (vectorial) representation of a spatial scene $e^{20,21}$, i.e. ensembles of egocentric boundary or object vector neurons encoding a point of view in craniotopic coordinates. The precuneus is traditionally viewed as medial continuation of lateral parietal area 7 (an area that exhibits activity related to eye-movements) and its central part (area $\mathrm{PGm}^{92}$ ) exhibits dense connectivity with the dorsolateral prefrontal cortex, suggesting involvement in higher cognitive processing, such as working memory. Interestingly, this part of the precuneus also receives inputs form the posterior-most portion of the precuneus, approximately congruent with area PO/V6A, which has strong connections with upstream visual areas. Hence, visual inputs posterior of the precuneus (and the retrosplenial cortex just anterior to it) may hold precursor representations for egocentric vector coding neurons. In primates, which have a greater reliance on vision, spatial view cells [G] ${ }^{93}$ have been found in the hippocampus, alongside place cells ${ }^{94}$. The former may be the product of a coordinate transform ${ }^{95}$, similar to that proposed for boundary and object vector cells.

In rodents, the medial surface that is opposite (lateral) posterior parietal areas is dominated by the retrosplenial cortex ${ }^{96}$, where egocentric boundary cells were found ${ }^{14,15}$. Thus, structures that provide 
input to the rodent retrosplenial cortex may similarly hold precursor representations for egocentric vector coding neurons. Connections have been reported between areas $7 \mathrm{~m} / \mathrm{ip}$ and the striatum (in primates $)^{97}$. A homologous connection could underlie the finding of egocentric boundary coding neurons in the striatum of rodents ${ }^{13}$, which could be used to inform cue-based striatal navigation.

\section{[H2] Non-perceptual information}

The emphasis on the sensory aspects of egocentric vectorial responses is complicated by the finding that some egocentric boundary coding neurons code for boundaries behind the animal ${ }^{13,14}$, outside visual and whisking range. For border and boundary vector cells this may be explained via learned associations with place cells, an account that applies also to 'trace' responses. If place cells have reciprocal connections to boundary vector cells and border cells, they can activate the allocentric boundary coding neurons with which they are associated via pattern completion $[\mathbf{G}]$ and guarantee orientation-independent firing outside the field of view. This can then translate to the egocentric frame of reference via a reference frame transformation ${ }^{21}$. The same mechanism may make boundary and object location information outside the field of view available during mental navigation $^{21,98,99}$, if path integration inputs from grid cells (driven by mock motor efference) can update place cell firing. That is, trace vector cells may be the consequence of vector coding coupled with pattern completion. This notion has two major implications. First, place cell firing should precede boundary vector cell firing during recall, which in turn should precede the firing of gainfield neurons. Gain-field neuron firing should then precede firing in egocentric boundary coding neurons with receptive fields behind the animal. Second, a lesion to the hippocampus, irrespective of induced memory deficits, could reduce egocentric boundary coding neurons to a purely sensory representation: that is, it could abolish 'trace' responses and activity for scene elements behind the animal.

\section{[H2] Functional differences between border, boundary, object and trace cells}

A major open question is what functional characteristics give rise to the different response properties of egocentric and allocentric border, boundary, trace and object vector cells. It is unclear how these cells differ at the levels of cyto-architecture, microcircuit anatomy and, above all, sensitivity to distinct environmental features or scene elements (see also Table 1).

Border cells respond like boundary vector cells but only for proximal boundaries, and do not respond to suspended objects (unlike object vector cells). Thus, it is possible that border cells are specifically tactile boundary vector cells. Trace vector cells respond like theoretical boundary 
vector cells, responding to any environmental feature (with larger features producing more firing than smaller ones), but with additional mnemonic, pattern completion properties. Object vector cells, on the other hand, respond only to objects internal to the environment and not to the surrounding boundaries. They also respond to larger objects but firing seems to concentrate towards the ends. As outlined above, in the absence of other factors differentiating internal objects from surrounding barriers this suggests that they have on-center-off-surround receptive fields sensitive to non-tactile inputs (e.g. from suspended objects).

These different cell types may have different uses, with some cells representing stable, extended environmental features which can more reliably support self-localisation (such as border cells resetting grid cell firing patterns ${ }^{33}$ ), while others represent more ephemeral, potentially mobile but behaviorally relevant local cues (such as object vector cells, or goal vector cells in bats). It is important to note that grid cells can also be used to calculate vectors between arbitrary locations $^{32,100-103}$, however, in this Review we have focused on neurons with explicitly vectorial tuning curves.

\section{[H1] Conclusions}

We have reviewed the current extent of experimental findings and theoretical work regarding neurons with vectorial receptive fields far from the sensory periphery. We have endeavoured to provide a functional taxonomy of the growing number of cell types that share a vectorial receptive field structure, yet exhibit important differences in their firing properties and anatomical loci. Table 1 summarizes this effort. Our current understanding of these neural responses and their role in spatial cognition is the result of close interplay between experiments and theoretical predictions that has allowed the allocentric spatial memory system in the hippocampal formation (and its egocentric inputs) to be elucidated at multiple levels ${ }^{104}$. The computational problem of self-localizing, memorizing that location and all the associated environmental features distributed around it, and making that information available in the future through memory recall in visuo-spatial imagery, can now be mapped onto our detailed knowledge of single cell responses. Nevertheless, major open questions mandate further experimental and theoretical work to refine and possibly reshape our growing understanding of spatial memory, and our understanding of what the hippocampal system does. Spatial relations are a subset of relational information ${ }^{105}$ and recent work shows how the extended hippocampal system may process non-spatial information ${ }^{106,107}$, potentially implying a radical generalisation of the role played by cells that exhibit receptive fields with vectorial character in spatial paradigms. 


\section{References}

1. O'Keefe, J. \& Dostrovsky, J. The hippocampus as a spatial map. Preliminary evidence from unit activity in the freely-moving rat. Brain Res. (1971) doi:10.1016/0006-8993(71)90358-1.

2. Hafting, T., Fyhn, M., Molden, S., Moser, M. B. \& Moser, E. I. Microstructure of a spatial map in the entorhinal cortex. Nature (2005) doi:10.1038/nature03721.

3. Taube, J. S., Muller, R. U. \& Ranck, J. B. Head-direction cells recorded from the postsubiculum in freely moving rats. I. Description and quantitative analysis. $J$. Neurosci. (1990) doi:10.1523/jneurosci.10-02-00420.1990.

4. Taube, J. S., Muller, R. U. \& Ranck, J. B. Head-direction cells recorded from the postsubiculum in freely moving rats. II. Effects of environmental manipulations. J. Neurosci. (1990) doi:10.1523/jneurosci.10-02-00436.1990.

5. Barry, C. et al. The boundary vector cell model of place cell firing and spatial memory. Reviews in the Neurosciences (2006) doi:10.1515/REVNEURO.2006.17.1-2.71.

6. Lever, C., Burton, S., Jeewajee, A., O’Keefe, J. \& Burgess, N. Boundary vector cells in the subiculum of the hippocampal formation. J. Neurosci. (2009) doi:10.1523/JNEUROSCI.1319-09.2009.

7. Solstad, T., Boccara, C. N., Kropff, E., Moser, M. B. \& Moser, E. I. Representation of geometric borders in the entorhinal cortex. Science (80-. ). (2008) doi:10.1126/science.1166466.

8. Savelli, F., Yoganarasimha, D. \& Knierim, J. J. Influence of boundary removal on the spatial representations of the medial entorhinal cortex. Hippocampus (2008) doi:10.1002/hipo.20511.

9. Deshmukh, S. S. \& Knierim, J. J. Influence of local objects on hippocampal representations: Landmark vectors and memory. Hippocampus (2013) doi:10.1002/hipo.22101.

10. Høydal, Ø. A., Skytøen, E. R., Andersson, S. O., Moser, M. B. \& Moser, E. I. Object-vector coding in the medial entorhinal cortex. Nature (2019) doi:10.1038/s41586-019-1077-7.

11. Taube, J. S. The Head Direction Signal: Origins and Sensory-Motor Integration. Annu. Rev. Neurosci. (2007) doi:10.1146/annurev.neuro.29.051605.112854.

12. Wang, C. et al. Egocentric coding of external items in the lateral entorhinal cortex. Science (80-. ). 362, 945-949 (2018).

13. Hinman, J. R., Chapman, G. W. \& Hasselmo, M. E. Neuronal representation of environmental boundaries in egocentric coordinates. Nat. Commun. (2019) doi:10.1038/s41467-019-10722-y. 
14. Alexander, A. S. et al. Egocentric boundary vector tuning of the retrosplenial cortex. Sci. Adv. (2020) doi:10.1126/sciadv.aaz2322.

15. Wijngaarden, J. B. G. van, Babl, S. S. \& Ito, H. T. Representation of Distance and Direction of Nearby Boundaries in Retrosplenial Cortex. bioRxiv (2019) doi:10.1101/807453.

16. Peyrache, A., Schieferstein, N. \& Buzsáki, G. Transformation of the head-direction signal into a spatial code. Nat. Commun. 8, (2017).

17. Gofman, X. et al. Dissociation between Postrhinal Cortex and Downstream Parahippocampal Regions in the Representation of Egocentric Boundaries. Curr. Biol. (2019) doi:10.1016/j.cub.2019.07.007.

18. Nakazawa, K. et al. Requirement for hippocampal CA3 NMDA receptors in associative memory recall. Science (80-. ). 297, 211-218 (2002).

19. O’Keefe, J. \& Nadel, L. Hippocampus Anatomy. Hippocampus as a Cogn. Map (1978).

20. Byrne, P., Becker, S. \& Burgess, N. Remembering the past and imagining the future: A neural model of spatial memory and imagery. Psychol. Rev. 114, 340-375 (2007).

21. Bicanski, A. \& Burgess, N. A neural-level model of spatial memory and imagery. Elife 7, 13 (2018).

22. Sarel, A., Finkelstein, A., Las, L. \& Ulanovsky, N. Vectorial representation of spatial goals in the hippocampus of bats. Science (80-. ). 355, 176-180 (2017).

23. O’ Keefe, J. \& Burgess, N. Geometric determinants of the place fields of hippocampal neurons. Nature (1996) doi:10.1038/381425a0.

24. Hartley, T., Burgess, N., Lever, C., Cacucci, F. \& O’Keefe, J. Modeling place fields in terms of the cortical inputs to the hippocampus. Hippocampus (2000) doi:10.1002/10981063(2000)10:4<369::AID-HIPO3>3.0.CO;2-0.

25. Burgess, N., Jackson, A., Hartley, T. \& O’Keefe, J. Predictions derived from modelling the hippocampal role in navigation. Biol. Cybern. (2000) doi:10.1007/s004220000172.

26. McNaughton, B., Knierim, J. \& Wilson, M. Vector Encoding and the Vestibular Foundations of Spatial Cognition: Neurophysiological and Computational Mechanisms. in The Cognitive Neurosciences (ed. Gazzaniga, M. S.) 585-595 (MIT Press, Boston, 1994).

27. Stewart, S., Jeewajee, A., Wills, T. J., Burgess, N. \& Lever, C. Boundary coding in the rat subiculum. Philos. Trans. R. Soc. B Biol. Sci. (2014) doi:10.1098/rstb.2012.0514.

28. Lever, C., Burgess, N., Cacucci, F., Hartley, T. \& O’Keefe, J. What can the hippocampal representation of environmental geometry tell us about Hebbian learning? Biol. Cybern. (2002) doi:10.1007/s00422-002-0360-z.

29. Grieves, R. M., Duvelle, É. \& Dudchenko, P. A. A boundary vector cell model of place field 
repetition. Spat. Cogn. Comput. 18, 217-256 (2018).

30. Lever, C., Wills, T., Cacucci, F., Burgess, N. \& O’Keefe, J. Long-term plasticity in hippocampal place-cell representation of environmental geometry. Nature (2002) doi:10.1038/416090a.

31. Bienenstock, E. L., Cooper, L. N. \& Munro, P. W. Theory for the development of neuron selectivity: Orientation specificity and binocular interaction in visual cortex. J. Neurosci. (1982) doi:10.1523/jneurosci.02-01-00032.1982.

32. Edvardsen, V., Bicanski, A. \& Burgess, N. Navigating with grid and place cells in cluttered environments. Hippocampus (2019) doi:10.1002/hipo.23147.

33. Hardcastle, K., Ganguli, S. \& Giocomo, L. M. Environmental Boundaries as an Error Correction Mechanism for Grid Cells. Neuron (2015) doi:10.1016/j.neuron.2015.03.039.

34. Keinath, A. T., Epstein, R. A. \& Balasubramanian, V. Environmental deformations dynamically shift the grid cell spatial metric. Elife (2018) doi:10.7554/eLife.38169.

35. Becker, S. \& Burgess, N. Modelling spatial recall, mental imagery and neglect. in Advances in Neural Information Processing Systems (2001).

36. Burgess, N., Becker, S., King, J. A. \& O'Keefe, J. Memory for events and their spatial context: Models and experiments. in Philosophical Transactions of the Royal Society B: Biological Sciences (2001). doi:10.1098/rstb.2001.0948.

37. Evans, T., Bicanski, A., Bush, D. \& Burgess, N. How environment and self-motion combine in neural representations of space. J. Physiol. 594, 6535-6546 (2016).

38. McNaughton, B.L., Battaglia, F.P., Jensen, O., Moser, E.I., Moser, M. B. Path integration and the neural basis of the 'cognitive map'. Nat. Rev. Neurosci. 7, 663-78 (2006).

39. O'Keefe, J. \& Burgess, N. Dual phase and rate coding in hippocampal place cells: Theoretical significance and relationship to entorhinal grid cells. Hippocampus (2005) doi:10.1002/hipo.20115.

40. Koenig, J., Linder, A. N., Leutgeb, J. K. \& Leutgeb, S. The spatial periodicity of grid cells is not sustained during reduced theta oscillations. Science (80-. ). (2011) doi:10.1126/science. 1201685 .

41. Berger, T. W., Swanson, G. W., Milner, T. A., Lynch, G. S. \& Thompson, R. F. Reciprocal anatomical connections between hippocampus and subiculum in the rabbit: Evidence for subicular innervation of regio superior. Brain Res. (1980) doi:10.1016/0006-8993(80)904631.

42. Köhler, C. Intrinsic projections of the retrohippocampal region in the rat brain. I. The subicular complex. J. Comp. Neurol. (1985) doi:10.1002/cne.902360407. 
43. Commins, S., Aggleton, J. P. \& O’Mara, S. M. Physiological evidence for a possible projection from dorsal subiculum to hippocampal area CA1. Exp. Brain Res. (2002) doi:10.1007/s00221-002-1158-x.

44. Shao, L. R. \& Dudek, F. E. Electrophysiological evidence using focal flash photolysis of caged glutamate that CA1 pyramidal cells receive excitatory synaptic input from the subiculum. J. Neurophysiol. (2005) doi:10.1152/jn.00877.2004.

45. Jackson, J. et al. Reversal of theta rhythm flow through intact hippocampal circuits. Nat. Neurosci. (2014) doi:10.1038/nn.3803.

46. Sun, Y. et al. Cell-type-specific circuit connectivity of hippocampal CA1 revealed through cre-dependent rabies tracing. Cell Rep. (2014) doi:10.1016/j.celrep.2014.02.030.

47. Xu, X., Sun, Y., Holmes, T. C. \& López, A. J. Noncanonical connections between the subiculum and hippocampal CA1. Journal of Comparative Neurology (2016) doi:10.1002/cne.24024.

48. Sun, Y., Nitz, D. A., Holmes, T. C. \& Xu, X. Opposing and complementary topographic connectivity gradients revealed by quantitative analysis of canonical and noncanonical hippocampal CA1 inputs. eNeuro (2018) doi:10.1523/ENEURO.0322-17.2018.

49. Sun, Y. et al. CA1-projecting subiculum neurons facilitate object-place learning. Nat. Neurosci. (2019) doi:10.1038/s41593-019-0496-y.

50. Poulter, S., Hartley, T. \& Lever, C. The Neurobiology of Mammalian Navigation. Current Biology (2018) doi:10.1016/j.cub.2018.05.050.

51. Langston, R. F. et al. Development of the spatial representation system in the rat. Science (80-. ). (2010) doi:10.1126/science.1188210.

52. Wills, T. J., Cacucci, F., Burgess, N. \& O'Keefe, J. Development of the hippocampal cognitive map in preweanling rats. Science (80-. ). (2010) doi:10.1126/science.1188224.

53. Chen, G., Lu, Y., King, J. A., Cacucci, F. \& Burgess, N. Differential influences of environment and self-motion on place and grid cell firing. Nat. Commun. (2019) doi:10.1038/s41467-019-08550-1.

54. Doeller, C. F. \& Burgess, N. Distinct error-correcting and incidental learning of location relative to landmarks and boundaries. Proc. Natl. Acad. Sci. U. S. A. (2008) doi:10.1073/pnas.0711433105.

55. Knierim, J. J., Lee, I. \& Hargreaves, E. L. Hippocampal place cells: Parallel input streams, subregional processing, and implications for episodic memory. Hippocampus (2006) doi:10.1002/hipo.20203.

56. Chersi, F. \& Burgess, N. The Cognitive Architecture of Spatial Navigation: Hippocampal 
and Striatal Contributions. Neuron (2015) doi:10.1016/j.neuron.2015.09.021.

57. Packard, M. G. \& McGaugh, J. L. Inactivation of hippocampus or caudate nucleus with lidocaine differentially affects expression of place and response learning. Neurobiol. Learn. Mem. (1996) doi:10.1006/nlme.1996.0007.

58. Knierim, J. J., Neunuebel, J. P. \& Deshmukh, S. S. Functional correlates of the lateral and medial entorhinal cortex: Objects, path integration and local - Global reference frames. Philosophical Transactions of the Royal Society B: Biological Sciences (2014) doi:10.1098/rstb.2013.0369.

59. LaChance, P. A., Todd, T. P. \& Taube, J. S. A sense of space in postrhinal cortex. Science (80-. ). (2019) doi:10.1126/science.aax4192.

60. Kunz, L. et al. A neural code of egocentric spatial information in human medial temporal lobe. bioRxiv (2020). doi:10.1101/2020.03.03.973131.

61. Jercog, P. E. et al. Heading direction with respect to a reference point modulates place-cell activity. Nat. Commun. (2019) doi:10.1038/s41467-019-10139-7.

62. Sharp, P. E. Computer simulation of hippocampal place cells. Psychobiology (1991) doi:10.3758/BF03327179.

63. Pouget, A. \& Sejnowski, T. J. Spatial transformations in the parietal cortex using basis functions. J. Cogn. Neurosci. (1997) doi:10.1162/jocn.1997.9.2.222.

64. Galletti, C., Battaglini, P. P. \& Fattori, P. Eye Position Influence on the Parieto-occipital Area PO (V6) of the Macaque Monkey. Eur. J. Neurosci. (1995) doi:10.1111/j.14609568.1995.tb01047.x.

65. Snyder, L. H., Grieve, K. L., Brotchie, P. \& Andersen, R. A. Separate body- and worldreferenced representations of visual space in parietal cortex. Nature 394, 887-891 (1998).

66. Salinas, E. \& Abbott, L. F. Transfer of coded information from sensory to motor networks. $J$. Neurosci. (1995) doi:10.1523/jneurosci.15-10-06461.1995.

67. Pouget, A., Deneve, S. \& Duhamel, J. R. A computational perspective on the neural basis of multisensory spatial representations. Nat. Rev. Neurosci. (2002) doi:10.1038/nrn914.

68. Burgess, N., Maguire, E. A., Spiers, H. J. \& O'Keefe, J. A temporoparietal and prefrontal network for retrieving the spatial context of lifelike events. Neuroimage (2001) doi:10.1006/nimg.2001.0806.

69. Zipser, D. A computational model of hippocampal place fields. Behav. Neurosci. (1985) doi:10.1037/0735-7044.99.5.1006.

70. Touretzky, D. S. \& Redish, A. D. Theory of rodent navigation based on interacting representations of space. Hippocampus (1996) doi:10.1002/(SICI)1098- 
1063(1996)6:3<247::AID-HIPO4>3.0.CO;2-K.

71. Recce, M. \& Harris, K. D. Memory for places: A navigational model in support of Marr's theory of hippocampal function. Hippocampus (1996) doi:10.1002/(SICI)10981063(1996)6:6<735::AID-HIPO15>3.0.CO;2-1.

72. Lambrey, S. et al. Distinct visual perspective-taking strategies involve the left and right medial temporal lobe structures differently. Brain (2008) doi:10.1093/brain/awm317.

73. Chrastil, E. R., Sherrill, K. R., Hasselmo, M. E. \& Stern, C. E. There and back again: Hippocampus and retrosplenial cortex track homing distance during human path integration. J. Neurosci. (2015) doi:10.1523/JNEUROSCI.1209-15.2015.

74. Epstein, R. A., Parker, W. E. \& Feiler, A. M. Where am i now? Distinct roles for parahippocampal and retrosplenial cortices in place recognition. J. Neurosci. (2007) doi:10.1523/JNEUROSCI.0799-07.2007.

75. Wang, C., Chen, X. \& Knierim, J. J. Egocentric and allocentric representations of space in the rodent brain. Curr. Opin. Neurobiol. 60, 12-20 (2020).

76. Tsao, A., Moser, M. B. \& Moser, E. I. Traces of experience in the lateral entorhinal cortex. Curr. Biol. (2013) doi:10.1016/j.cub.2013.01.036.

77. Weible, A. P., Rowland, D. C., Pang, R. \& Kentros, C. Neural correlates of novel object and novel location recognition behavior in the mouse anterior cingulate cortex. J. Neurophysiol. (2009) doi:10.1152/jn.00214.2009.

78. Weible, A. P., Rowland, D. C., Monaghan, C. K., Wolfgang, N. T. \& Kentros, C. G. Neural correlates of long-term object memory in the mouse anterior cingulate cortex. J. Neurosci. (2012) doi:10.1523/jneurosci.5265-11.2012.

79. Deshmukh, S. S. \& Knierim, J. J. Representation of non-spatial and spatial information in the lateral entorhinal cortex. Front. Behav. Neurosci. (2011) doi:10.3389/fnbeh.2011.00069.

80. O'Keefe, J. Place units in the hippocampus of the freely moving rat. Exp. Neurol. (1976) doi:10.1016/0014-4886(76)90055-8.

81. Poulter, S., Lee, S. A., Dachtler, J., Wills, T. J. \& Lever, C. Vector Trace cells in the Subiculum of the Hippocampal formation. bioRxiv (2019) doi:10.1101/805242.

82. Andersen, R. A. Multimodal integration for the representation of space in the posterior parietal cortex. in Philosophical Transactions of the Royal Society B: Biological Sciences (1997). doi:10.1098/rstb.1997.0128.

83. Clark, B. J., Simmons, C. M., Berkowitz, L. E. \& Wilber, A. A. The retrosplenial-parietal network and reference frame coordination for spatial navigation. Behav. Neurosci. (2018) doi:10.1037/bne0000260. 
84. Burwell, R. \& Agster, K. Learning and Memory: A Comprehensive Reference. (2008).

85. Burwell, R. D., Witter, M. P. \& Amaral, D. G. Perirhinal and postrhinal cortices of the rat: A review of the neuroanatomical literature and comparison with findings from the monkey brain. Hippocampus (1995) doi:10.1002/hipo.450050503.

86. Nitz, D. A. Tracking route progression in the posterior parietal cortex. Neuron (2006) doi:10.1016/j.neuron.2006.01.037.

87. Alexander, A. S. \& Nitz, D. A. Retrosplenial cortex maps the conjunction of internal and external spaces. Nat. Neurosci. (2015) doi:10.1038/nn.4058.

88. Wilber, A. A., Clark, B. J., Forster, T. C., Tatsuno, M. \& McNaughton, B. L. Interaction of egocentric and world-centered reference frames in the rat posterior parietal cortex. $J$. Neurosci. (2014) doi:10.1523/JNEUROSCI.0511-14.2014.

89. Julian, J. B., Ryan, J., Hamilton, R. H. \& Epstein, R. A. The Occipital Place Area Is Causally Involved in Representing Environmental Boundaries during Navigation. Curr. Biol. (2016) doi:10.1016/j.cub.2016.02.066.

90. Shine, J. P., Valdés-Herrera, J. P., Tempelmann, C. \& Wolbers, T. Evidence for allocentric boundary and goal direction information in the human entorhinal cortex and subiculum. Nat. Commun. (2019) doi:10.1038/s41467-019-11802-9.

91. Crowne, D. P., Richardson, C. M. \& Dawson, K. A. Parietal and frontal eye field neglect in the rat. Behav. Brain Res. (1986) doi:10.1016/0166-4328(86)90067-7.

92. Margulies, D. S. et al. Precuneus shares intrinsic functional architecture in humans and monkeys. Proc. Natl. Acad. Sci. 106, 20069-20074 (2009).

93. Rolls, E. T., Robertson, R. G. \& Georges-François, P. Spatial view cells in the primate hippocampus. Eur. J. Neurosci. (1997) doi:10.1111/j.1460-9568.1997.tb01538.x.

94. Courellis, H. S. et al. Spatial encoding in primate hippocampus during free navigation. PLoS Biol. (2019) doi:10.1371/journal.pbio.3000546.

95. Rolls, E. T. Spatial coordinate transforms linking the allocentric hippocampal and egocentric parietal primate brain systems for memory, action in space, and navigation. Hippocampus (2019) doi:10.1002/hipo.23171.

96. Burwell, R. D. \& Amaral, D. G. Cortical afferents of the perirhinal, postrhinal, and entorhinal cortices of the rat. J. Comp. Neurol. (1998) doi:10.1002/(SICI)10969861(19980824)398:2<179::AID-CNE3>3.0.CO;2-Y.

97. Cavada, C. \& Goldman-Rakic, P. S. Multiple visual areas in the posterior parietal cortex of primates. Prog. Brain Res. 95, 123-137 (1993).

98. Horner, A. J., Bisby, J. A., Zotow, E., Bush, D. \& Burgess, N. Grid-like processing of 
imagined navigation. Curr. Biol. (2016) doi:10.1016/j.cub.2016.01.042.

99. Bellmund, J. L., Deuker, L., Navarro Schröder, T. \& Doeller, C. F. Grid-cell representations in mental simulation. Elife 5, (2016).

100. Erdem, U. M. \& Hasselmo, M. A goal-directed spatial navigation model using forward trajectory planning based on grid cells. Eur. J. Neurosci. 35, 916-931 (2012).

101. Erdem, U. M. \& Hasselmo, M. E. A biologically inspired hierarchical goal directed navigation model. J. Physiol. Paris (2014) doi:10.1016/j.jphysparis.2013.07.002.

102. Kubie, J. L. \& Fenton, A. A. Linear look-ahead in conjunctive cells: An entorhinal mechanism for vector-based navigation. Front. Neural Circuits (2012) doi:10.3389/fncir.2012.00020.

103. Bush, D. \& Burgess, N. A Hybrid Oscillatory Interference/Continuous Attractor Network Model of Grid Cell Firing. J. Neurosci. (2014) doi:10.1523/JNEUROSCI.4017-13.2014.

104. Marr, D. C. \& Poggio, T. From understanding computation to understanding neural circuitry. Neurosci. Res. Program Bull. (1977).

105. Eichenbaum, H. What versus where: Non-spatial aspects of memory representation by the hippocampus. in Current Topics in Behavioral Neurosciences (2018). doi:10.1007/7854_2016_450.

106. Constantinescu, A. O., OReilly, J. X. \& Behrens, T. E. J. Organizing conceptual knowledge in humans with a gridlike code. Science (80-. ). 352, 1464-1468 (2016).

107. Whittington, J. C. et al. The Tolman-Eichenbaum Machine: Unifying space and relational memory through generalisation in the hippocampal formation. bioRxiv (2019) doi:10.1101/770495.

108. Alexander, A. S. et al. Egocentric boundary vector tuning of the retrosplenial cortex. bioRxiv 702712 (2019) doi:10.1101/702712.

109. Hafting, T., Fyhn, M., Molden, S., Moser, M.-B. \& Moser, E. I. Microstructure of a spatial map in the entorhinal cortex. Nature 436, 801-806 (2005).

110. Fyhn, M., Hafting, T., Treves, A., Moser, M.-B. \& Moser, E. I. Hippocampal remapping and grid alignment in entorhinal cortex. Nature 446, 190-194 (2007).

111. Buxbaum, L. J. et al. Hemispatial neglect: Subtypes, neuroanatomy, and disability. Neurology (2004) doi:10.1212/01.WNL.0000113730.73031.F4.

112. Bisiach, E. \& Luzzatti, C. Unilateral Neglect of Representational Space. Cortex (1978) doi:10.1016/S0010-9452(78)80016-1.

113. Coslett, H. B. Neglect in vision and visual imagery: A double dissociation. Brain (1997) doi:10.1093/brain/120.7.1163. 
114. Ortigue, S. et al. Pure representational neglect after right thalamic lesion. Ann. Neurol. (2001) doi:10.1002/ana.1139.

\section{Acknowledgements}

$\mathrm{AB}$ and NB acknowledge funding by the ERC Advanced grant NEUROMEM and a Wellcome Principal Research Fellowship to NB. We thank Daniel Bush for useful discussions feedback on the manuscript.

\section{Author contributions}

The authors contributed equally to all aspects of the article.

\section{Competing interests}

The authors declare no competing interests.

\section{Publisher's note}

Springer Nature remains neutral with regard to jurisdictional claims in published maps and institutional affiliations.

Table 1: Experimentally characterised and theoretically modelled properties of vector-coding neurons

\begin{tabular}{|l|l|l|l|l|c|c|c|c|}
\hline Cell type & $\begin{array}{c}\text { Anatomica } \\
\mathbf{l} \\
\text { location }\end{array}$ & $\begin{array}{c}\text { Reference } \\
\text { frame }\end{array}$ & $\begin{array}{c}\text { Distance } \\
\text { tuning }\end{array}$ & $\begin{array}{c}\text { Object } \\
\text { selectivity }\end{array}$ & $\begin{array}{c}\text { Fir for } \\
\text { objects/boun } \\
\text { daries } \\
\text { outside } \\
\text { FOV? }\end{array}$ & $\begin{array}{c}\text { Long term } \\
\text { memory } \\
\text { traces? }\end{array}$ & $\begin{array}{c}\text { Experi } \\
\text { mental } \\
\text { refs }\end{array}$ & $\begin{array}{c}\text { Theoretica } \\
\text { I } \\
\text { refs }\end{array}$ \\
\hline $\begin{array}{l}\text { Boundary } \\
\text { vector cells } \\
\text { (BVCs) }\end{array}$ & Subiculum & Allocentric & Spectrum & Spectrum\# & Yes & $?$ & $5,6,27$ & $5,20,21,24,29,35$ \\
\hline $\begin{array}{l}\text { Border } \\
\text { cells }\end{array}$ & mEC & Allocentric & Proximal & Spectrum\# & $?$ & $?$ & 7 & 32,34 \\
\hline $\begin{array}{l}\text { Landmark } \\
\text { vector cells }\end{array}$ & HPC (CA1) & Allocentric & $?$ & $\begin{array}{l}\text { Internal } \\
\text { objects }\end{array}$ & Yes & $?$ & 9 & 107 \\
\hline $\begin{array}{l}\text { Object } \\
\text { vector cells } \\
\text { OVCs) }\end{array}$ & mEC & Allocentric & Spectrum & $\begin{array}{l}\text { Internal } \\
\text { objects }\end{array}$ & Yes & $?$ & 10 & 21,107 \\
\hline $\begin{array}{l}\text { Trace } \\
\text { vector cells }\end{array}$ & Subiculum & Allocentric & Spectrum & Spectrum & Yes & Yes & 81 & $21 \dagger$ \\
\hline $\begin{array}{l}\text { Egocentric } \\
\text { boundary }\end{array}$ & $\begin{array}{l}\text { RSC, PC, } \\
\text { STM, }\end{array}$ & Egocentric & Spectrum & Spectrum\# & Yes & $?$ & $12,13,17,10$ & $20,21,35$ \\
\hline
\end{tabular}




\begin{tabular}{|c|c|c|c|c|c|c|c|c|}
\hline cells & POR, LEC & & & & & & & \\
\hline $\begin{array}{l}\text { Egocentric } \\
\text { BCs }\end{array}$ & RSC & Egocentric & Proximal & Spectrum\# & ? & $?$ & 15 & None \\
\hline $\begin{array}{l}\text { Egocentric } \\
\text { OVCs }\end{array}$ & LEC & Egocentric & Spectrum & $\begin{array}{l}\text { Internal } \\
\text { objects\$ }\end{array}$ & Yes & ? & 12 & 21 \\
\hline $\begin{array}{l}\text { Head } \\
\text { direction } \\
\text { gain } \\
\text { modulated } \\
\text { BVCs }\end{array}$ & $\begin{array}{l}\text { POR, PaS, } \\
\text { dPrS, mEC }\end{array}$ & both & Spectrum & Spectrum\# & Yes & $?$ & 17 & $20,21,35$ \\
\hline $\begin{array}{l}\text { Head } \\
\text { direction } \\
\text { gain } \\
\text { modulated } \\
\text { OVCs }\end{array}$ & ? & both & ? & $\begin{array}{l}\text { Internal } \\
\text { objects\$ }\end{array}$ & Yes\$ & $?$ & None & 21 \\
\hline $\begin{array}{l}\text { Anchor (or } \\
\text { Center- } \\
\text { bearing) } \\
\text { cells }\end{array}$ & POR,PHC & Egocentric & Spectrum & $\begin{array}{l}\text { No object } \\
\text { selectivity }\end{array}$ & Yes & $?$ & 59,60 & None \\
\hline
\end{tabular}

Note that for cell types whose firing is modulated by head direction it cannot be decided if their boundary related firing is allocentric or egocentric. dPrS, dorsal presubiculum; FOV, field of view; HPC, hippocampus; IEC, lateral entorhinal cortex; mEC, medial entorhinal cortex; PaS, parasubiculum; PC, parietal cortex; PHC, (human) parahippocampal cortex; POR, postrhinal cortex; RSCm retrosplenial cortex; STM, striatum; . Question marks indicate a lack of experimental data. $\dagger$ BVCs and OVCs in this modeling study have the additional property of being trace vector cells. \# Theoretical receptive field is independent of object size (larger objects produce larger response fields), but experiments with small objects are lacking; \$ Theoretical definition only, experiments are lacking.

Fig. 1| General properties of vectorial receptive fields a| The top panels show firing rate maps of a place cell and a grid cell, recorded as a rodent forages in a square box; warm colours represent high firing rates. In the lower panels, black circles illustrate the allocentric receptive fields of these neurons: when the animal occupies these locations the neuron fires ${ }^{1,109} . \mathbf{b} \mid$ Examples of vector-coding cell firing patterns. The top row shows the firing rate map of a boundary vector cell (left) and an illustration of the receptive field of this cell, which is located at a fixed distance and direction from the agent ${ }^{6}$ (middle). The right panel indicates the vector pointing from agent location to the receptive field. When the receptive field is occupied by a boundary, the neuron fires. The bottom row shows the firing rate map of an object vector cell (left; white circles indicate objects) and an illustration of the receptive field of this cell, which is located at a fixed distance and direction from the agent ${ }^{10}$ (middle) The right panel indicates the vector pointing from agent location to the receptive field. When the receptive field is occupied by an object (black discs), the neuron fires. $\mathbf{c |}$ Multiple vector-coding neurons are hypothesised to form a grid of receptive fields (grey circles) around an agent (black arrowhead indicates the direction that the agent is facing). Neurons fire (indicated by yellow shading) if their receptive fields are occupied by a boundary (yellow bar in this example). The physical distribution of neurons in the brain need not match the topology of their receptive fields. $\mathbf{d} \mid$ 
Arranging the neurons in part $\mathbf{c}$ as a two-dimensional sheet in which their location corresponds to the position of their receptive fields reveals the approximate shape of the boundary, encoded by the active neurons across that sheet. The neurons are agnostic about boundary identity: any environmental feature in the receptive field elicits firing. Part a is adapted, with permission, from Refs. ${ }^{51,110}$, Creative Commons 4 licence. Top left panel in part $\mathrm{b}$ is adapted, with permission from top from Ref. ${ }^{6}$. Bottom left panel in part $\mathrm{b}$ is adapted, with permission from Ref. ${ }^{10}$.

Fig. 2| Allocentric vector coding neurons Several types of allocentric vector coding neurons have been identified. a| The firing rate map (right) of a boundary vector cell located in the subiculum, as a rodent explores an arena (left, recoding surface (black) and walls (orange), surrounded by a curtain) into which a barrier has been inserted. Insertion of the barrier led to duplication of firing fields (as indicated by higher levels of activity when the animal is above the lower wall and above the barrier. I.e. the cell fire regardless of boundary identity. $\mathbf{b} \mid$ In an experiment with an elevated arena, small traversable gaps in the base of the arena also elicit boundary vector cell activity (see second band of activity in bottom firing rate map), as do the large drops at the edges of the arena. $\mathbf{c}$ The receptive field size of boundary vector cells varies with their distance from the agent, with larger receptive fields for more distant locations. $\mathbf{d} \mid$ Border cells in the medial entorhinal cortex only fire for nearby boundaries, including any barriers inserted into the arena. I.e. they resemble close range boundary vector cells in c. el. Firing rate maps of two object vector cells recorded in the medial entorhinal cortex as a rodent explores a circular arena (white circles indicate objects). The cells exhibit firing fields at fixed allocentric distance and direction, irrespective of object identity, and do not fire for extended boundaries (cf. k). $\mathbf{f} \mid$ The existence of object vector cells was proposed in a model of spatial cognition as the basis of object-location memory ${ }^{21}$. The left panel shows the simulated environment used in this study, containing three objects (green), and the agent's trajectory. The right panel shows firing rate map of a simulated object vector cell, which exhibits firing when the animal is at a fixed allocentric distance and direction from the object, irrespective of object identity. The red dashed line is provided for comparison with the firing rate map . g) The top panel, polar plot illustrating the angular and distance tuning of a large sample of object vector cells. Each dot represents one object vector cell. The bottom panel illustrates the distance tuning of the same population of cells. There is an approximately uniform coverage of angles; however, fewer object vector cells have firing fields at large distances from the agent. h) Left and centre panels are firing rate maps of two concurrently recorded object vector cells. The cells maintain their relative vectorial differences (angle and distance from an object) when the animal is placed in a new environment (room A versus room B). The right panels show polar plots of a single head-direction (HD) cell in both environments, indicating their tuning to specific angles. The angular difference (compare polar plots) matches that of the object vector fields. i| Object vector cells continue to respond to object after they have been elevated. $\mathbf{j} \mid$ Firing rate maps of two landmark vector cells in the hippocampus, recorded as a rodent explores a square box containing multiple distinct objects. Cells exhibit firing when the animals are at fixed allocentric 
directions and distances from an object but their firing is modulated by object identity in a complex manner (they do not fire exclusively for a single object nor are these cells object agnostic. k) Comparison of the receptive fields proposed to underlie the properties of object vector cells and boundary vector cells. The 'oncentre/off-surround' object vector cell receptive fields could explain their lack of response to extended boundaries, which would drive both excitation and inhibition of the cell. Black triangle represents the agent, green bar a boundary, green square an object. Parts a-c are adapted, with permission, from Ref. ${ }^{6}$. Part d adapted, with permission, from Ref. ${ }^{7}$. Parts e,h-j adapted, with permission, from Ref. ${ }^{10}$. Part $\mathrm{f}$ is adapted, with permission, from Ref. ${ }^{21}$. Part $\mathrm{g}$ is adapted, with permission, from Ref. ${ }^{9}$.

\section{Fig. 3| Egocentric vector coding cells.}

Several types of vector coding neurons with egocentric receptive fields have recently been identified. a|. An example of ecogentric coding. The left panel shows the trajectory (grey line) of an animal as it explores a square arena (grey line) and the locations at which firing occurred in an egocentric boundary cell in the stiratum (dots, color-coded according to the animal's direction of movement at the time of firing ${ }^{13}$. The red circle and black arrow show the animal's location and heading direction when one selected spike was fired. Middle panel: the boundary positions (red lines) relative to the animal when this spike was fired are plotted in an egocentric reference frame in which the animal is considered to be stationary at the centre of the plot. The right panel shows the firing rate map of the cell in egocentric coordinates, created by plotting the frequency of egocentric boundary locations across all spikes as a proportion of time with that boundary occupancy. I.e. how often does an egocentric boundary location coincide with an emitted spike relative to the time that boundary was present at those egocentric coordinates.. This reveals the egocentric vectorial receptive field of the cell. $\mathbf{b} \mid$ Two examples of striatal egocentric boundary cells. Left panel, color-coded spikes show that firing was restricted to a given movement direction for each wall (same color coding as in a). The egocentric firing rate map (second panel) shows firing for proximate walls on the left of the animal. The second example (panels 3-4), shows another egocentric boundary cell, which has a large egocentric receptive field located behind the animal. $\mathbf{c} \mid$ A retrosplenial egocentric boundary cell with a proximal receptive field loses its firing when the walls of the recording arena are removed. Distal fields persist when walls are removed (not shown). d| Retrosplenial egocentric boundary cells fire independently of environmental shape, shown here in a circular and a square arena. e| The left column shows object-related (Voronoi) partitioning of a rectangular arena ${ }^{12}$ to enable the analysis of neural firing of an egocentric object bearing neuron in lateral entorhinal cortex. Black circles indicate objects. The color-coding of the recording arena indicates the Rayleigh vector (that is, the mean vector length ${ }^{22}$ ) of the egocentric bearing tuning curve (warm colors indicate sharper tuning), calculated at each location in the environment. The right column shows the relationship between the firing of the cell and the egocentric bearing of the 4 objects (top to bottom) relative to the animal. Shaded regions indicate common bearing, suggesting the cell fires regardless of the object's identity whenever an object is found at a given egocentric bearing. $\mathbf{f} \mid$ Panel 1 (top left) shows the spiking locations of a postrhinal cortex cell as the animal explores a square arena, color-coded by head 
direction and superimposed on the animal trajectory. Line plots show the relationship between the firing rate of the cell and the center-bearing (panel 2), center-distance (panel 3), and head direction (panel 4). Parts a and $\mathrm{b}$ are adapted, with permission, from Ref. ${ }^{13}$. Parts $\mathrm{C}$ and $\mathrm{D}$ are adapted from Ref. ${ }^{14}$. Part e Adapted from Ref. $^{12}$. Part F Adapted from Ref. ${ }^{59}$

\section{Figure 4: Reference frame transformations}

a| The egocentric-allocentric 'gain-field' transformation circuit, showing responses to a boundary (but equally applicable to objects $)^{21}$. The left shaded box illustrates the properties of 4 egocentric boundary cells (eBCs in the parietal cortex. The central panel shows 16 gain-field neurons in the retrosplenial transformation circuit (RSC), with connectivity to each of the eBCs indicated by matching symbols (stars, hexagons, triangles or squares). The rightmost box indicates 4 different possible head directions (coded for by head direction cells in the thalamus). The lower box shows boundary vector cells (BVCs, e.g. in subiculum). Light grey arrows indicate connection to and from retrosplenial cortex. Example firing rate maps for each cell are shown in a simple square environment. In each case, firing related to the North, East, South and West walls is depicted in blue, yellow, purple and red, respectively. The receptive fields of BVCs and eBCs are indicated by dashed ovals around the agent (black triangles). In bottom-up mode (that is, during perception and/or encoding), HD-driven gain modulation (dark grey arrows) ensures that the activity of a given eBC drives different RSC cells depending on the agent's HD. In the example shown, the eBC that signals a boundary 'ahead' drives different RSC neurons (green arrows) depending on head direction. E.g. the eBC signalling a boundary ahead will contact the RSC cells markerd with the green star at different HDs. These RSC cells then drive different BVC mapping the eBC activity for a boundary 'ahead' to different allocentric directions (nominally North, South etc.). That is inputs to BVCs are selected from the RSC cells shown in the column above each BVC, due to the HD gain modulation. In top-down mode (during imagery or recall), egocentric BCs are driven by RSCs (green arrows from RSC to parietal cortex), which are in turn driven by BVCs. $\mathbf{b} \mid$ Experimental evidence confirms the theoretical prediction of boundary coding neurons modulated by head direction ${ }^{17}$, found in the parasubiculum, dorsal presubiculum, medial entorhinal and postrhinal cortices of rats. The left panel shows the trajectory of a rodent with the locations at which a neuron fired indicated (red dots). The second panel shows the resultant firing rate map. Splitting the date by by head direction (panel 3) reveals the gain modulation. I.e. the cell fires only for North $(\mathrm{N})$. The fourth panel shows the head direction selectivity of the cell, again indicating maximum firing for North (polar plot; the high amplitude and narrow tuning indicates tight directional specificity). The fifth panel shows the cell's egocentric rate map $($ ahead $=u p)$ and the sixth panel a polar plot of firing rates for boundaries at different egocentric angles (polar plot). Part a is adapted, with permission, from Ref. ${ }^{21}$. Part $b$ is adapted, with permission, from Ref. $^{17}$. 
Figure 5: Trace cells and models of spatial cognition a| A schematic of the so-called BB-model of spatial cognition ${ }^{21}$ in which sensory inputs support egocentric (boundary and object) vector cell firing (e.g. in parietal cortex). The retrosplenial cortex uses gain-modulation by head direction to perform the transformation from egocentric to allocentric coding, yielding allocentric vector cells. Place cells (PCs) representing location, and perirhinal neurons representing the identities of scene elements complete the medial temporal representations. Place cells binding allocentric vector cells (bidirectional arrow) allow for location estimates based on egocentric inputs (via the intermediate coordinate transfrom). Perirhinal cells are assumed to be driven by ventral visual stream inputs (not shown). b| According to this model, top-down connections can lead to trace responses for missing scene elements. In the example shown, the red rectangle indicates the position of a barrier in the square environment that has previously been encoded but is now absent. Trace activity manifests in the population snapshot of egocentric boundary cell firing and allocentric BVC firing. Each panel shows the firing rates of populations of neurons arranged according to the topology of their receptive fields (cf. Figure 1D). Hebbian associations from place cells reactivate the allocentric (and thence egocentric via the coordinate transformation, cf. Figure 4A) representations for the encoded boundary configuration around the animal. $\mathbf{c} \mid$ At the single cell level, traces of scene elements manifest as trace fields in the firing rate map for the non-existent boundary (left panel). Right panel: illustration of the receptive field of the BVC. d| Non-vectorial trace responses have been reported in the lateral entorhinal ${ }^{76}$. In this study it was shown that a cell will develop a trace field at the location of an object that is repeatedly teleported (black square: original position of the object, blue squares: subsequent positions of the object). However, no vectorial relationship was reported. E) In another study, a hippocampal cell develops a non-vectorial trace field at the location of a newly inserted object (star shape, introduced in session 5) that is subsequently removed (in session 6) (white circles indicate stable objects). A-C adapted from Ref. ${ }^{21}$. Part d adapted from Ref. $^{76}$. Part e Adapted from Ref. ${ }^{9}$.

\section{BOX 1: Vectorial receptive fields, neglect and human spatial cognition}

The idea, derived from rodent experiments, that the receptive fields of vector coding neurons form arrays that cover external space is consistent with the effects of primate pathologies. In humans, hemispatial neglect commonly manifests following a right-sided stroke (usually resulting from the rupture of a prominent blood vessel above the right parietal cortex). The phenomenology reported by afflicted patients is composed of various deficits in attention, perception (known as perceptual neglect) and mnemonic function relating to objects in the field of view contralateral to the injury (left), despite the presence of intact visual areas ${ }^{111}$. This suggests that perceived stimuli on that side of the field of view are not processed appropriately or not at all. Representational neglect describes a subset of these symptoms ${ }^{112}$. For example, one individual affected by representational neglect was unable to visualize (imagine) a familiar environment (the town square in Milan) in its entirety (lacking the left hemifield, contra-lateral to the lesion), but could imagine of all locations around the square by adopting different egocentric points of view. This phenomenology can be accounted for by a family of computational models that presuppose that an array of neurons with egocentric receptive 
fields in the parietal cortex ${ }^{20,21,35,63}$ holds an egocentric perceptual representation of the current point of view and can instantiate the output of the egocentric-allocentric reference frame transformation (which takes place in the retrosplenial cortex, see also FIG. 4 and FIG. 5). A lesion (red colored cell bodies) that removes the left half of these egocentric cells removes the ability to perceive or reconstruct a full representation of the environment (see the figure, left column), but leaves the underlying viewpoint invariant memory (the allocentric representation) intact ${ }^{112}$. It is unknown how far the reconstruction during recall is propagated back to lower level sensory cortices (question mark in bottom row). In rare cases, representational neglect can occur without perceptual neglect ${ }^{113,114}$. This is accounted for by the above models if the lesion affects the neurons responsible for the egocentric-allocentric reference frame transformation but leaves parietal areas intact (see the figure, right column). In this case, perception is unimpaired, but recall in visuo-spatial imagery is subject to neglect, as indicated by white cell bodies (lacking input but nominally intact). Abbreviations: MTL, medial temporal lobe; RSC, retrosplenial cortex.

\section{Receptive field}

A limited area of 'stimulus space' that drives a neuron to fire when occupied by a stimulus.

Stimulus space can be sensory (e.g. a patch of skin) or abstract (e.g. a location in an environment).

Grid cells

Cells in entorhinal cortex with spatial receptive fields arranged in repeating hexagonal patterns across the environment, thought to underlie path integration and vector navigation.

\section{Head direction cells}

Cells that fire when an animal's head is at a specific angle relative to external landmarks, found in an extended network of cortical and subcortical brain areas.

\section{Spatial cognition}

The capacity of human and non-human animals for mental representation and manipulation of spatial information.

Firing rate maps

Histograms of time-averaged neural activity binned according to the location of the animal in an experimental arena. 
Path integration

Tracking changes in self-location by integrating self-motion information such as linear and angular velocity.

Firing field

A patch of elevated firing in a firing rate map. Note, one receptive field can generate multiple firing fields as the agent moves to bring stimuli into the receptive field.

Reference frame

A common coordinate system in which to relate multiple observations.

Theta oscillations

Prominent 5-10 Hz oscillations in the local field potential, ubiquitous in rodents during locomotion and, in shorter bursts, associated with memory in humans.

Competitive learning

A learning algorithm that assumes winner-take-all dynamics in the post-synaptic population (e.g. via lateral inhibition), such that only one post-synaptic neuron is active enough to strengthen its connections from active pre-synaptic neurons.

\section{Gain-field neurons}

Neurons whose firing rate in response to the presence of a stimulus in its receptive field is gainmodulated by a second signal. E.g. the eye-position can up- or down-modulate the response to a stimulus in a retinal receptive field.

\section{Hebbian Learning}

The learning theory originated by Donald Hebb in 1949, stating that a neuron that partakes in making another neuron fire will strengthen its synapse to that neuron (regardless of individual spike timings).

Spatial view cells

Cells in the primate hippocampus that fire whenever a given location in an environment is observed, regardless of the animal's location and pose in that environment. 


\section{Pattern completion}

The reactivation of all neurons comprising a pre-existing pattern of neural activity causes by activity in a subset of those neurons. 


\section{| PERCEPTUAL NEGLECT \\ REPRESENTATIONAL NEGLECT (pure)}

SENSORY

PARIETAL/EGO

LEFT HS : RIGHT HS

CORTICES

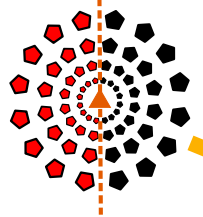

RSC/EGO-ALLO TRANSFORMATION

LEFT HS | RIGHT HS

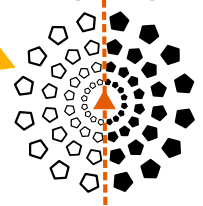

\section{SENSORY} CORTICES

PARIETAL/EGO LEFT HS : RIGHT HS

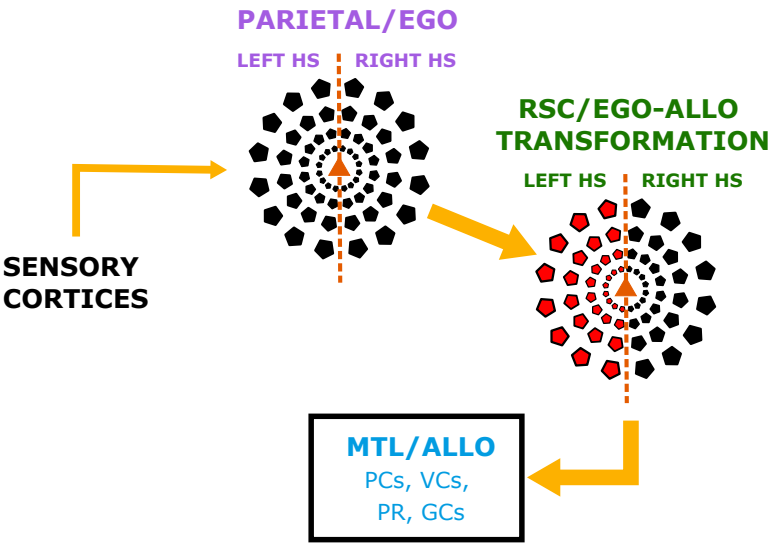

\section{PARIETAL/EGO}

LEFT HS : RIGHT HS

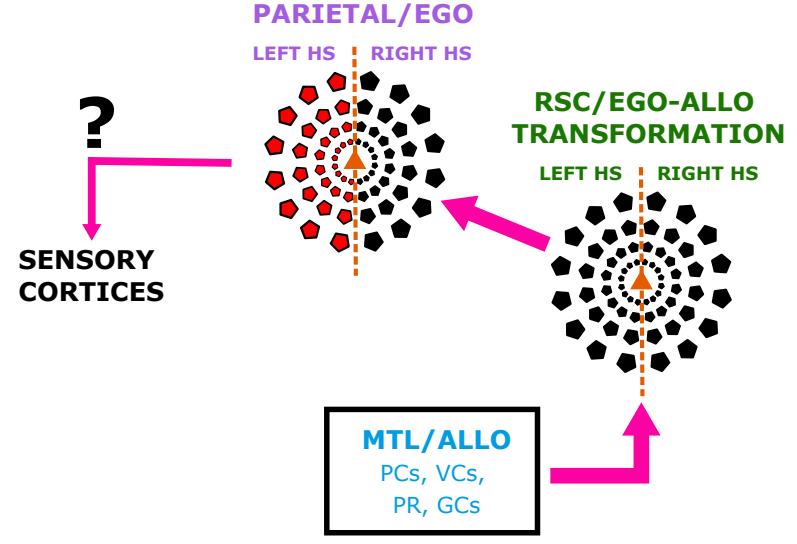

\section{PARIETAL/EGO}

LEFT HS : RIGHT HS

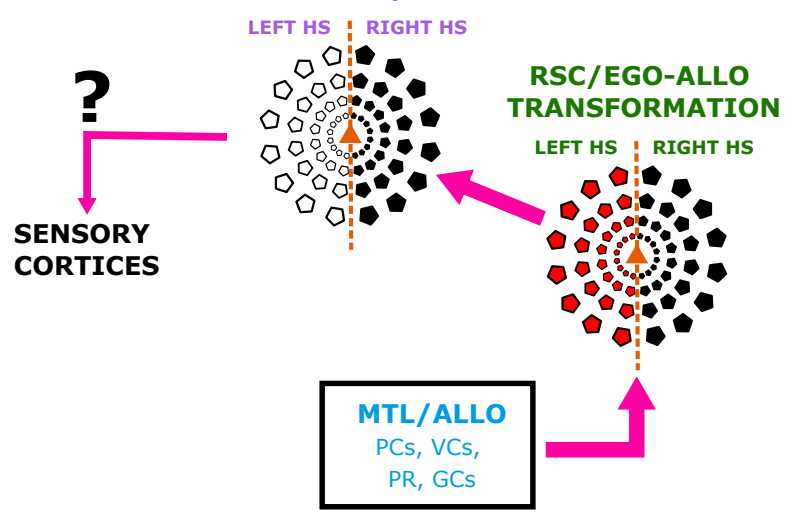

\section{Lesioned cells}

Cells lacking input from lesioned upstream cells 


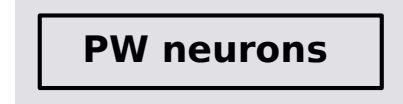

\section{Egoc. Allocentric \\ RF pos. Rate Map}

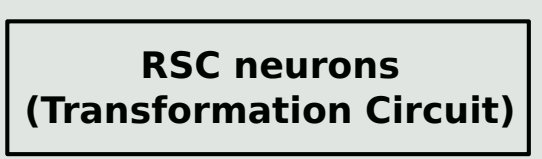

\begin{tabular}{|c|}
\hline HD \\
modulation
\end{tabular}
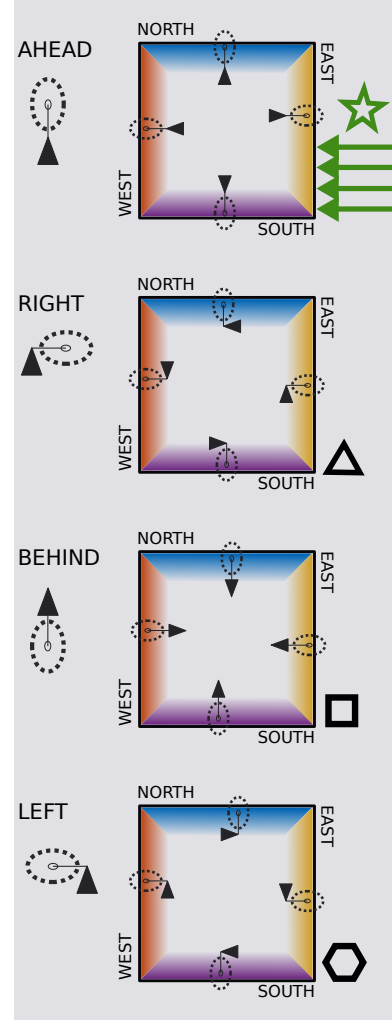

\section{Boundary Vector Cells}
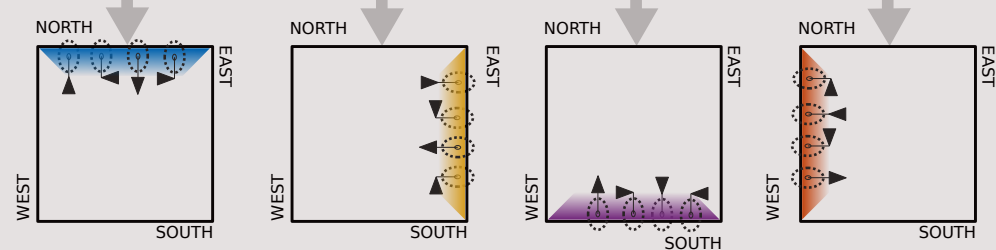

Allocentric

Rate Map

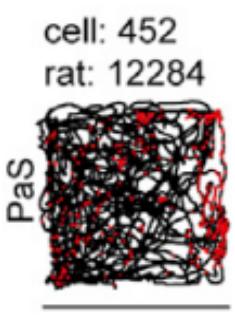

$150 \mathrm{~cm}$.

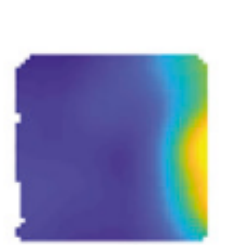

$5 \mathrm{~Hz}$

$(1-5 \mathrm{~Hz})$

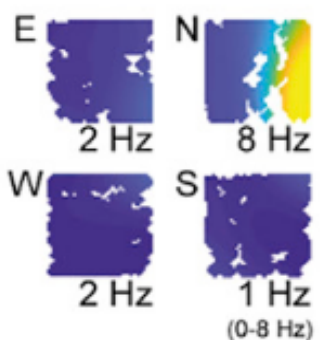

Rayleigh: 0.59

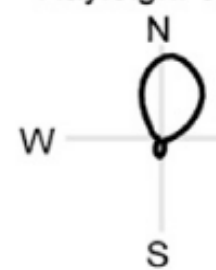

E

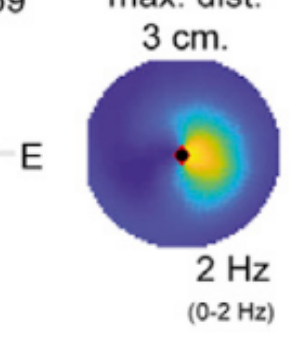

Rayleigh: 0.4

$\mathrm{F}$

L

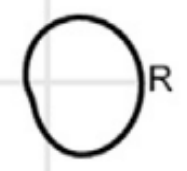

B
HD EAST

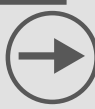

HD WEST

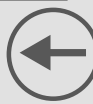




\section{Egocentric boundary cells}

A Construction of egocentric firing rate maps

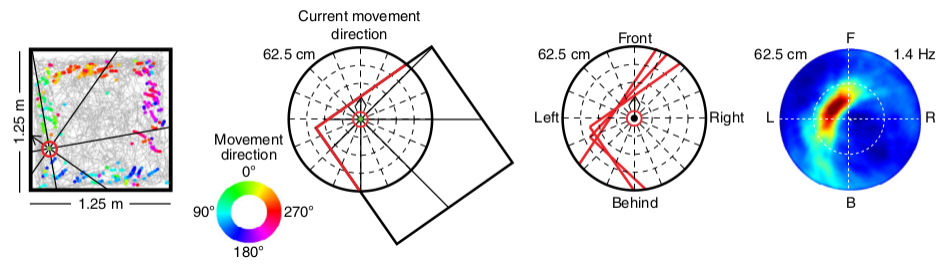

D Proximal and distal egocentric receptive fields.

Distal field is behind the animal.
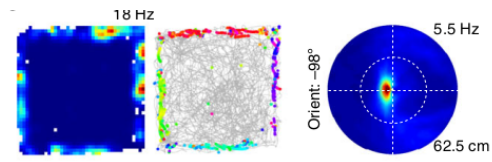

C

Without walls proximal EBC firing fields disappear
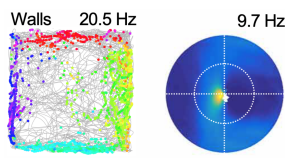

Walls $2.3 \mathrm{~Hz}$

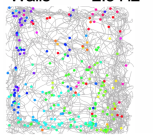

$0.5 \mathrm{~Hz}$

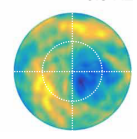

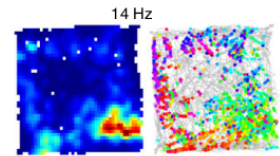

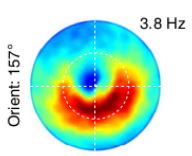

Egocentric object bearing cells E $\begin{aligned} & \text { Firing for the egocentric } \\ & \text { bearing of local objects }\end{aligned}$
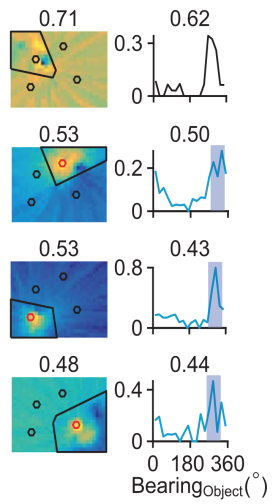

\section{Center distance} and bearing cells
EBC firin
agnostic

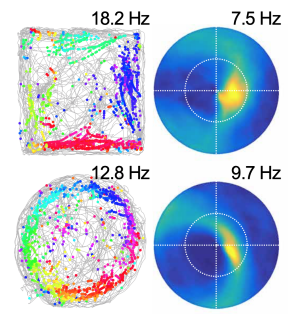

Center distance and bearing correlations

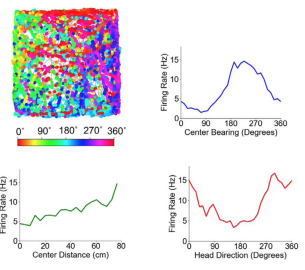




\section{Boundary vector cells}

$\Delta \quad$ Firing independent of boundary identity

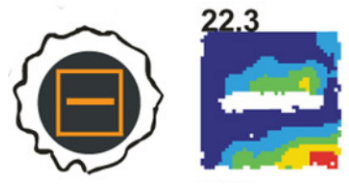

B

Firing in response to gaps or drops
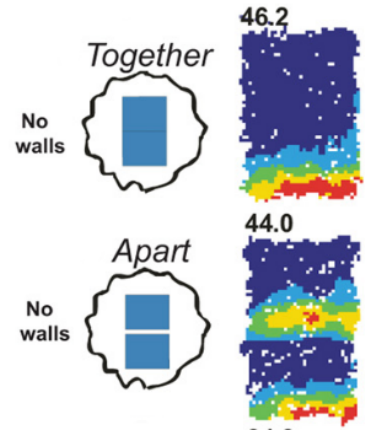

Receptive field size varies with distance

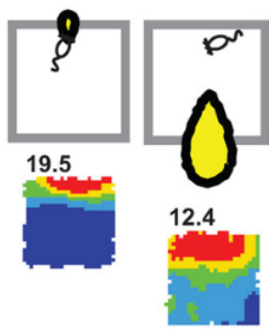

\section{Border cells}

D Firing independent of boundary identity

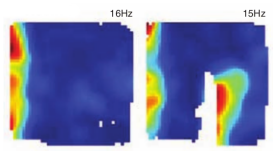

\section{Object vector cells}

다ring independent of object identity
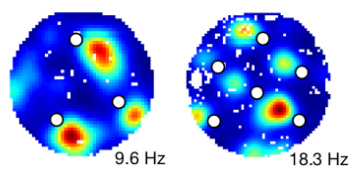

E Theoretically predicted object vector cells
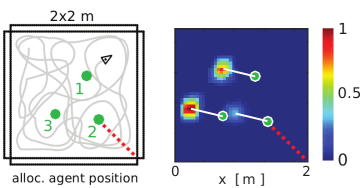

H

Allocentric angles shift in register between environments
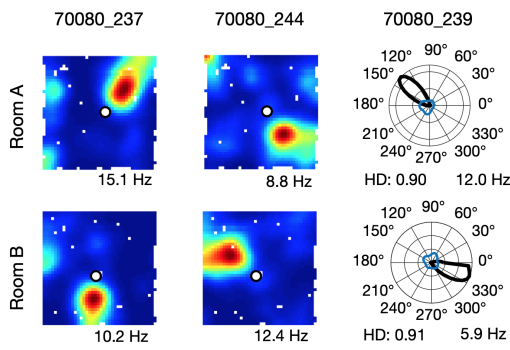

HD: $0.90 \quad 12.0 \mathrm{~Hz}$
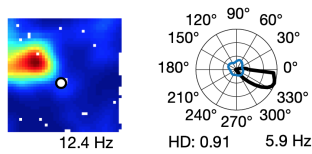

Distribution of allocentric angles and distances
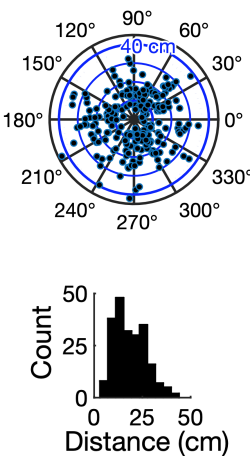

1 Firing in response to elevated objects

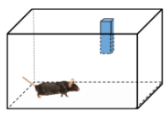

Suspended object

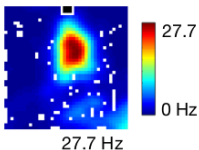

\section{Landmark vector cells}

Jiring relative to select objects

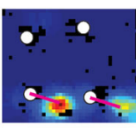

Unit 1

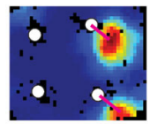

Unit 2

\section{Receptive field structure}

1 Possible relationship between BVC and OCV receptive fields

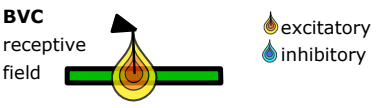

ovc

proposed receptive field

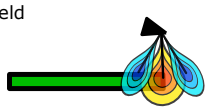

firing at ends

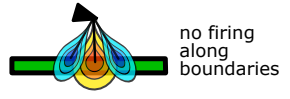
along boundaries

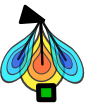

firing for small objects 
C

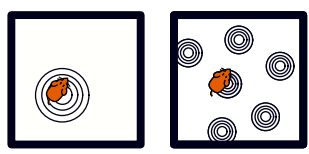

B

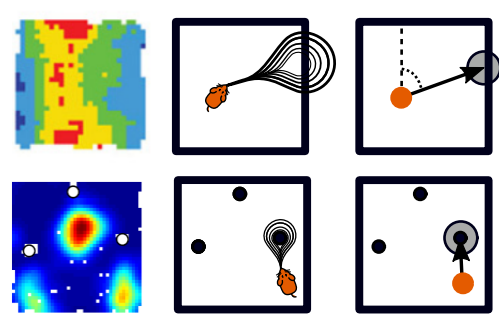

Array of receptive

fields in external

space, anchored

to an agent

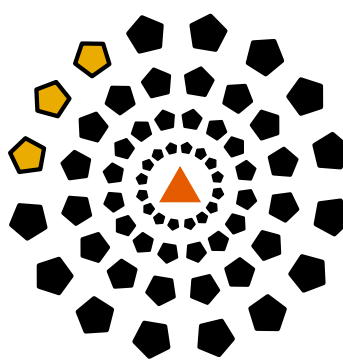

Neuronal activity arranged according to the topology of their receptive field 